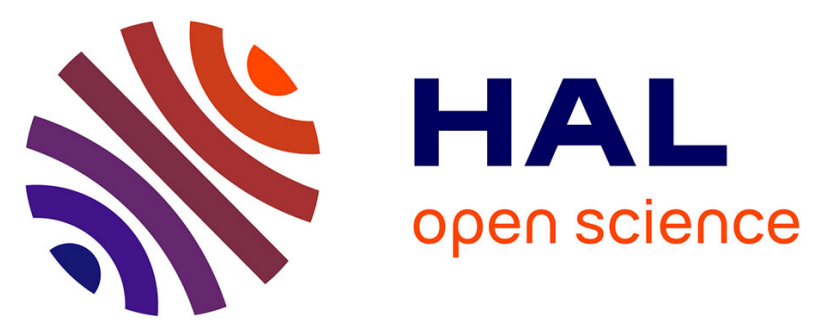

\title{
A prognostic function for complex systems to support production and maintenance co-operative planning based on an extension of object oriented Bayesian networks
}

Xavier Desforges, Mickaël Diévart, Bernard Archimède

\section{- To cite this version:}

Xavier Desforges, Mickaël Diévart, Bernard Archimède. A prognostic function for complex systems to support production and maintenance co-operative planning based on an extension of object oriented Bayesian networks. Computers in Industry, 2017, vol. 86, pp. 34-51. 10.1016/j.compind.2017.01.002 . hal-01490597

\author{
HAL Id: hal-01490597 \\ https://hal.science/hal-01490597
}

Submitted on 15 Mar 2017

HAL is a multi-disciplinary open access archive for the deposit and dissemination of scientific research documents, whether they are published or not. The documents may come from teaching and research institutions in France or abroad, or from public or private research centers.
L'archive ouverte pluridisciplinaire HAL, est destinée au dépôt et à la diffusion de documents scientifiques de niveau recherche, publiés ou non, émanant des établissements d'enseignement et de recherche français ou étrangers, des laboratoires publics ou privés. 


\section{Open Archive Toulouse Archive Ouverte (OATAO)}

OATAO is an open access repository that collects the work of Toulouse researchers and makes it freely available over the web where possible.

This is an author-deposited version published in: http://oatao.univ-toulouse.fr/ Eprints ID: 17506

To link to this article: DOI:10.1016/j.compind.2017.01.002

http://dx.doi.org/10.1016/j.compind.2017.01.002

\section{To cite this version:}

Desforges, Xavier and Diévart, Mickaël and Archimède, Bernard A prognostic function for complex systems to support production and maintenance co-operative planning based on an extension of object oriented Bayesian networks. (2017) Computers in Industry, vol. 86. pp. 34-51. ISSN 0166-3615 


\title{
A prognostic function for complex systems to support production and maintenance co-operative planning based on an extension of object oriented Bayesian networks
}

\author{
Xavier Desforges ${ }^{\mathrm{a}, *}$, Mickaël Diévart ${ }^{\mathrm{b}}$, Bernard Archimède $^{\mathrm{a}}$ \\ a Université Fédérale Toulouse Midi-Pyrénées, INPT, ENIT, Laboratoire Génie de Production, 65016 Tarbes, France \\ ${ }^{\mathrm{b}}$ Aéroconseil, 31703 Blagnac, France
}

Keywords:

Prognostics

Complex systems

Availability assessment

Preventive maintenance

Production planning

\begin{abstract}
A B S T R A C T
The high costs of complex systems lead companies to improve their efficiency. This improvement can particularly be achieved by reducing their downtimes because of failures or for maintenance purposes. This reduction is the main goal of Condition-Based Maintenance and of Prognostics and Health Management. Both those maintenance policies need to install appropriate sensors and data processes not only to assess the current health of their critical components but also their future health. These future health assessments, also called prognostics, produce the Remaining Useful Life of the components associated to imprecision quantifications. In the case of complex systems where components are numerous, the matter is to assess the health of whole systems from the prognostics of their components (the local prognostics). In this paper, we propose a generic function that assesses the future availability of complex systems from their local prognostics (the prognostics of their components) by using inferences rules. The results of this function can then be used as decision support indicators for planning productive and maintenance tasks. This function exploits a proposed extension for Object Oriented Bayesian Networks (OOBN) used to model the complex system in order to assess the probabilities of failure of components, functions and subsystems. The modeling of the complex system is required and it is presented as well as modeling transformations to tackle some OOBN limitations. Then, the computing inference rules used to define the future availability of complex systems are presented. The extension added to OOBN consists in indicating the components that should first be maintained to improve the availabilities of the functions and subsystems in order to provide a second kind of decision support indicators for maintenance. A fictitious multi-component system bringing together most of the structures encountered in complex systems is modeled and the results obtained from the application of the proposed generic function are presented as well as ways that production and maintenance planning can used the computed indicators. Then we show how the proposed generic prognostic function can be used to predict propagations of failures and their effects on the functioning of functions and subsystems.
\end{abstract}

\section{Introduction}

To improve their competitiveness in ever changing markets, companies need flexibility and responsiveness. This leads them to implement production equipment of goods or services ever more flexible, more responsive and therefore more complex but also more costly. With such production resources, the major challenge is to maintain them in operational condition with the highest level of availability for the lowest cost. The implementation of the

\footnotetext{
* Corresponding author.

E-mail address: xavier.desforges@enit.fr (X. Desforges).
}

Condition-Based Maintenance (CBM) and of Prognostic and Health Management (PHM) recommendations usually leads to the improvement of the equipment availability and the reduction of maintenance costs $[18,20,36]$. Indeed, CBM is the use of machinery run-time data to determine the machinery condition, which can be used to schedule required repair and maintenance prior to breakdown. PHM, which refers specifically to the phase involved with predicting future behavior, including the Remaining Useful Life (RUL) assessment, in terms of current operating state and with the scheduling of required maintenance actions to maintain system health, now enriches CBM $[28,44]$. The assessment of the RUL of components of a machinery is in fact the major issue of PHM. That is why PHM can also be implemented to guarantee the 
availability of assets, which is a typical demand in some ProductService Systems (PSS) whose business core is to provide machine capability rather than product ownership. Indeed, PHM enables to avoid unscheduled downtimes and contract penalties in PSS [37].

In the domain of PHM, many works contribute to assess more accurately the Remaining Useful Life (RUL) before the failure, which is also called time to failure, of a critical component of a system $[23,36]$. This mainly consists in assessing, with a given probability, the duration of use of a component before it reaches a level of degradation beyond which the risk of failure is too high [44]. This is shown in Fig. 1 where $t_{0}$ is the current duration of use. Three main approaches are developed [15]: experience-based prognostics, model-based prognostics and data-driven prognostics [4]. The experience-based approach uses data gathered from the experience feedback to identify reliability laws. The model-based approach is based on mathematical models of the physics of degradations of components [16]. The data-driven approach consists in transforming the monitoring data provided by the sensors installed on the system into reliable behavioral models of degradations [14]. Many works aim at assessing the RULs of components or at improving the accuracy of the prognostics for many kinds of components: ball-bearings $[28,43]$, gear trains $[48,49]$, train pantographs [17], braking systems [10], batteries $[13,19]$, etc., but also to predict crack growth in structures [31,33].

However, only the RULs of critical components are assessed because they require sensors and data processing resources to detect failure precursors and to estimate the remaining durations of use before the degradations reach the failure thresholds which correspond to the levels of degradation beyond which the risks of failure are too high [34]. In the absence of the RUL of a component, data such as MTTF (Mean Time To Failure) or MTBF (Mean Time Between Failures) can be used [34]. In this case, the RUL is calculated by subtracting the MTTF or the MTBF from the duration of use. RULs are estimates determined from predictions and MTTFs and MTBFs are often obtained statistically. Therefore those quantities are not only scalar and they are so associated to confidence or imprecision indicators listed in [34]. That is why most of the works dealing with the prognostics of components contribute to the assessment of the RULs as well as the definitions of their Probability Density Functions (PDFs) [32].

Although these previous works dealing with prognostics are component oriented, the implementation of CBM and PHM also requires the health assessment of the whole systems as well as decision supports for maintenance planning $[24,45]$. Muller et al. in
[29] propose the deployment of a prognosis process within an emaintenance architecture. This integration into the e-maintenance architecture is done element by element and provides a decision support for maintenance planning from the health conditions of the components but it does not assess the overall ability of the system to perform the future tasks. Voisin et al. in [45] define a generic prognosis business process but they do not describe the process that combines the RULs and their imprecisions in order to provide the prognosis of the system although they mention its interests. A more integrated approach has been developed in $[26,27]$. It consists of a method to model both the system of interest and the maintenance system thanks to Probabilistic Relational Models (PRM) that are used to choose the best maintenance strategy thanks to simulations that assesses key performance indicators.

Other works also consider the production management system such as the ones presented in $[1,9]$ that propose decision supports based on the health assessment of the systems. They requires the assessment of the risk that the systems will fail in fulfilling the operations the production planning assigns to them. This risk of failure is an input of the decision support for maintenance and production planning. Such decision supports are extended to industry to perform the maintenance activities at the better time [8]. Indeed, if knowing current and future health conditions of components is necessary to plan maintenance, knowing the ability of a system to perform future tasks is also necessary for production scheduling in order to provide a better compromise to satisfy the respective objectives of the maintenance system and of production management system $[5,6,35]$. Indeed, maintenance and production can plan conflicting activities on the resources they share: the machines while optimizing their own key performance indicators but may not optimize more global performance indicators [6]. Whereas maintenance determines the best practices to apply to components to set the productive technical systems at a desired availability level, production is more interested in the availability of functions of these same systems during the fulfillment of productive tasks it plans. Indeed a productive task does not necessary require the availability of all the functions of the productive system to be fulfilled. Thus productive systems can be exploited in a degraded mode (with one or maybe more unavailable functions) for some tasks while waiting for the best moment for their maintenance. This is the idea understood by "the capacity of the machine to perform the activity" mentioned in [6]. Examples are numerous: such as a five axis machine tool that can

Probability density of failure Degradation level at a given degradation level

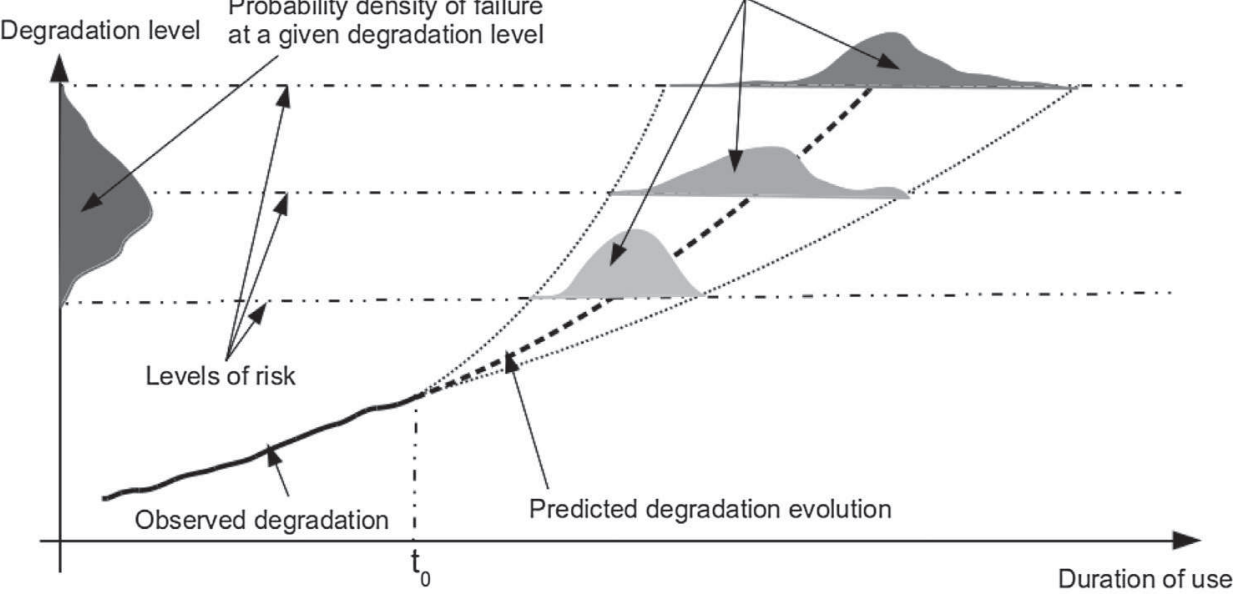

Fig. 1. Probability densities associated to RUL [44]. 
be used as a four axis machine tool and only for dry machining operations if its lubrication system is also failed; a truck with a failed cooling system cannot carry frozen food but it can carry food whose temperature does not need to be controlled. In this context, knowing the future availability of functions of the productive technical system according to the productive tasks to plan is as important as knowing the future health of its components to organize their maintenance.

However, in the field of prognostics, on one hand works deal with the assessments of RULs of components or structures and with the assessments of their accuracies or with the improvement of their accuracies. On the other hand, works deal with the planning of maintenance actions and production tasks from the prognostics of systems. However, as far as we know, no research work deals with the prognostic of complex systems from the prognostics of their components and/or their structures to provide the decision support indicators for the planning of maintenance actions and of production tasks. Nevertheless we notice works in which the modeling of the system for its future reliability assessment is directly use to define the best maintenance policy $[26,27,30]$. We also notice one work in which the success the future planned tasks flights is taken into account [9]. The systems (aircrafts) are considered as sets of line replaceable modules (components) for which RULs are known. An aircraft is considered as failed as soon as one of its line replaceable modules fails. If these considerations are convenient to test an optimization method for CBM, they are not relevant in terms of health assessment of the complex system that an aircraft is.

That is why, this paper presents a generic function based on Object Oriented Bayesian Networks (OOBN) to which an ability is added. The function provides decision support indicators satisfying the needs of maintenance and production planning, thanks to local prognostics and statistical data about unmonitored components such as Times To Failure (TTFs) and Times Between Failures (TBFs) and inferences. To implement this function, the complex system must be modeled. This requires the identification of the different relationships that can exist between the components of complex systems and also between the components and the functions they implement. Then, the paper presents the computation rules based on Bayesian networks to which we propose an extension. These rules are used to infer, at different levels, the probabilities that the components, functions, subsystems and the complex system will fail during the fulfillment of the operations the production planning assigns to it from the local prognostics. The proposed extension consists in defining the components that should first undergo maintenance in order to reduce the risk of failure during the fulfillment of the future operations as the definition of "intelligent prognostics" suggests it in [24]. Modeling transformations are necessary to process the proposed function; they are presented. They enable to tackle some OOBN limitations. To illustrate those rules, a fictitious multi-component system is presented. It brings together most of the structures encountered in complex systems. This example show results from different values of local prognostics and to show how the indicators computed by the proposed prognostic function could be exploited by maintenance and production planning. An emerging ability of the prognostic function that consists in predicting the impacts of failures onto the whole system is presented too as well as a way to use it in the case of consistency-based diagnostics. Finally, conclusions and prospects are listed.

\section{Modeling complex systems for their prognoses}

Complex systems are multi-component systems in which components may or may not interact with each other, with human beings and with their environments [50]. As the aim is the prognostics of complex systems and as it is very difficult to predict future behaviors of human beings and of the whole environment of a technical system, the notion of complex system is thus limited to multi-component systems with complex structures such as they are defined in [30]. The particular case of systems of systems in which properties can emerge is not considered.

Systems engineering addresses the design of complex systems that meet specified functions and performances at lower costs [22]. Therefore the implementation of a prognostic function in a system must be considered at the design stage [12]. This implementation requires knowledge about the system: the structural knowledge, the functional knowledge and the behavioral knowledge except the knowledge about the prognostic process [45]. In consistence with the standard ISO 13381-1, discrete models are more adapted for complex systems. More often, the degradation levels are represented with different states by using formalisms such as Markov chains and Bayesian networks. These states are generally defined by a physical reality whereas the transitions between states occur stochastically [16]. Discrete models were successfully implemented in the domain of prognostics for RUL assessment $[10,19,25,28,43]$. However the combination of the possible states for each of the numerous components of the complex systems makes the Markov chain models unmanageable [46]. Hence, we propose a discrete modeling based on Bayesian networks in which transitions between states of entities of the system are stochastic and whose values may impact the values of transitions between the states of other entities.

\subsection{Functional knowledge modeling}

In systems engineering, systems are considered from several points of view. One of them is the hierarchical view which breaks down a system into subsystems, then into functions, then into multiple levels of sub-functions till components implementing one or more sub-functions [22]. Knowing the abilities of functions of a complex system to perform future operations or their risks of failing while fulfilling them supports decision making for production and maintenance planning. Indeed, production planning knows the operations it assigns to complex systems and thus the functions that will be solicited and how long their solicitations will last. All operations assigned to the system do not necessarily solicit all its functions the same way and some may not be solicited at all. So, if a function of a system is not able to perform a task or to fulfill a sequence of tasks at an acceptable level of risk according to its prognostic, production and maintenance can jointly make a decision according to performance indicators. Possible decisions are: the system is assigned to another task that will not solicit the "risky" function or will solicit it less, the system is stopped for maintenance, the number of operations in the sequence is reduced and the maintenance of components that are likely to fail is planned ... The functional knowledge modeling aims at providing the sets of entities that implement the functions of the complex system. Those entities are either components or functions. This leads to a recursive definition of a function. At the lower levels of the hierarchical breakdown, one or several components implement the functions. Component are here considered as atomic entities on which the maintenance can act. They can be simple parts like joints or software modules but also more complex devices like servo-drives. At higher levels of the hierarchical breakdown, functions can be made of components and/or functions. Eventually, at the highest level, the subsystems gather functions. Thus, the systems engineering process enables to collect the necessary knowledge for functional modeling.

If an entity belonging to function fails or, in a more general way, becomes unable to provide the service for which it has been 
designed, the function to which it participates will also become unable to provide its service. Therefore, a function can only provide its service only if all the entities belonging to it are able to do so. Such functions are then called "simple functions". If functions of the system are or become unable to provide their services whereas their services will not be solicited for the planned productive tasks, the system can be exploited in the degraded mode that corresponds to the unavailability of those functions.

Simple functions become weak in terms of reliability when they are made of many entities, sub-entities, etc. That is why, sets of entities that provide a same service or a same function are often implemented to match the reliability or safety requirements [11]. These sets are redundant and can be made of only one entity. In those sets if one entity at least becomes unable to perform its own service, the whole set will not be able to perform the function it was designed for; but the other sets can still perform this function. However, if there is only one set that is able to perform the function, there is no more redundancy and, in many cases, the system must not begin a new task or operation mainly because of safety reasons. That is why two situations must be considered and their probabilities of occurrence must be assessed: the one for which none of redundant sets is able to ensure and the one for which only one set is able to ensure the function. For such cases, the functional modeling proposal consists in gathering the redundant sets or entities into what is then called a "redundancy function" in order to assess both the probabilities of loss of the function and of loss of redundancy. A redundancy function can be solicited by planned productive tasks in a degraded mode that corresponds to sets that are or become unable to provide the service if the probabilities of loss of the function and of loss of redundancy are still acceptable until the fulfilment of the productive tasks.

A graphical representation of the functional knowledge is presented in Fig. 2.

\subsection{Structural knowledge modeling}

The structural modeling aims at representing the direct interactions between entities (components or functions) and their failure modes mainly in order to propagate their effects [47]. Failure Modes and Effects Analysis (FMEA), fault trees or HAZard and OPerability (HAZOP) studies enable to collect the necessary knowledge for structural modeling. Indeed, those studies enable to identify what happens to other components or to functions when

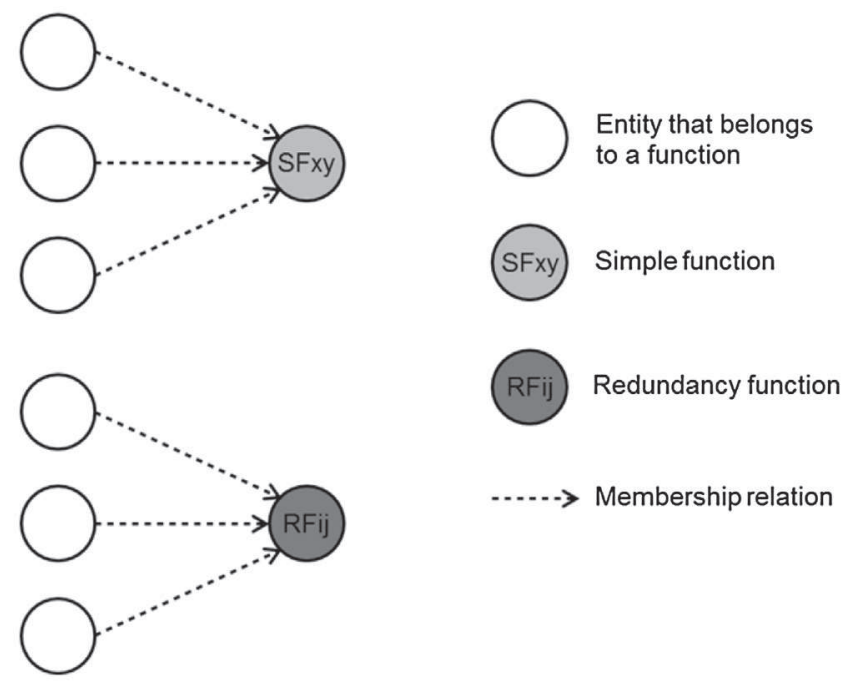

Fig. 2. Elements of functional knowledge modeling. one or several components fail [7]. Models used for system design can also be exploited such as SADT (Structured Analysis and Designed Technique) diagrams [26], or as SysML (Systems Modeling Language) diagrams like the internal blocks diagrams, activity diagrams, in which material, energy and data flows that are used, produced, transformed and exchanged by functions and components are represented. Therefore, this modeling consists in representing causality relationships between entities of the system that is, nevertheless, considered as functional modeling in [29].

A graphical representation of the structural knowledge is presented in Fig. 3. In this representation, the causal relationship means that the downstream entity will fail or will become out of order if the upstream entity fails or becomes out of order. Thus, only direct causal relationships must be modeled to avoid to consider a same event like several independent ones. Indeed, according to the structural model of Fig. 3, if E2 fails or becomes out of order, E5 will fail or will become out of order and then, consequently, E6 and E7 will fail or will become out of order too.

\subsection{Behavioral knowledge modeling}

The behavioral modeling mainly aims at defining the dynamical behavior of a system. Behavioral models are used to detect degradations and to analyze their trends in order to prognose the monitored components. The techniques of data acquisition and of data processing implemented in order to detect the degradations and to analyze their trends to define the RULs (with confidence or uncertainty indicators) of the components, are numerous and they also depend on the components to prognose [36].

The behavioral modeling also requires design knowledge of components, functions or subsystems. Many stakeholders are involved in the design of complex systems. They can design and provide simple parts or subsystems. The behavioral modeling may need that the supplier of a component provides its behavioral model to another partner. This may lead to the disclosure the know-how of the component supplier. Therefore, it would be more appropriate that suppliers also provide the prognostic systems of their components for their different failure modes. Indeed, they know the behavioral models and they can so implement the most relevant techniques. In this case, a supplier can provide either one prognostic for each mode of failure of the component or one prognostic for all its failure modes In this last case, the component is assumed having only one failure mode. These prognostics are here considered as local prognostics which are the inputs of the proposed prognostic function of complex systems. Consequently, a component has one or several local prognostics whatever its complexity is. Thus, local prognostics are considered as attributes of components. These associations between local prognostics and components constitute the behavioral knowledge modeling for the prognostic function.

A graphical representation of the behavioral knowledge is presented in Fig. 4.

In the particular case of redundancy functions, the redundant entities or sets of entities achieving the same service must firstly be gathered in order to create the redundancy function that

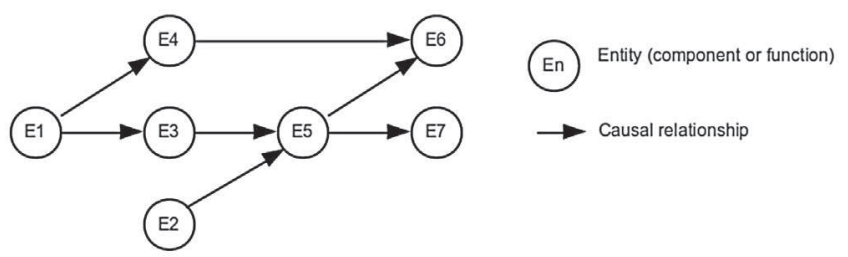

Fig. 3. Elements of structural knowledge modeling. 


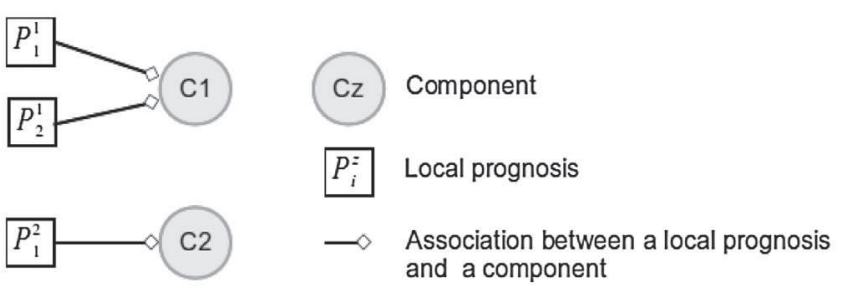

Fig. 4. Elements of behavioral knowledge modeling.

implement this service. Then, all the entities that contribute to the upper level service are gathered into a simple function. To illustrate this, we consider the example of the flight control structure presented in [11]. The flight control provides the references to the servo-actuators of the control surfaces of modern commercial aircrafts. In this paper, the flight control service (FCS) is implemented by three flight controllers (FC), two sticks (ST) one for each pilot in the cockpit, two rudders (RU) one for each pilot in the cockpit and three Air Data and Inertial Reference Units (AD) and we also consider that it requires power supply (PS). For the proposed generic function that provides decision support indicators for production and maintenance planning, the FCS must be modeled as shown in Fig. 5 where the prefix RF stands for redundancy function.

However implementing prognostic systems for all the components of complex systems would be too costly. Thus, data such as MTTF or MTBF and their imprecisions can be used [34] in the absence of the RULs of components as mentioned in the introduction. The data obtained from the MTTFs and MTBFs and the durations of use are also considered as local prognostics.

The elements of knowledge modeling that are presented in Figs. 2 and 3 show that the modeling of a complex system for implementing the generic function this paper propose has a directed graph structure. The directed graph $\boldsymbol{G}$ modeling a complex system is an ordered pair $G=(N, A)$ where $\boldsymbol{N}$ is the set of nodes and A is the set of unweighted $\operatorname{arcs}$. The set of $\operatorname{arcs} A$ is such as $A=$ $\left\{(E i, E j) \in N^{2} \mid E i \neq E j\right\}$ where $E i$ is the head and $E j$ is the tail. The set $\boldsymbol{N}$ is made of three kinds of nodes: components, simple functions and redundancy functions. Those three types of nodes for which their states are different, the computation of the probabilities of transitions between their states are also different makes OOBN suitable tool to implement the prognostic function. Indeed, OOBNs, thanks to the object oriented approach, enable to consider several types (named classes in the object oriented approach) with different attributes and methods to compute them [3]. Concepts, like specialization and polymorphism, are very helpful to ease the implementation of algorithms especially for graph traversal process with different classes of nodes. The OOBNs are extended by PRMs mainly to handle non-Bayesian uncertainties. PRMs also enable to implement recurrent patterns by classes of objects of interests as proposed in [26,27]. As the proposed approach is less integrated than in $[26,27]$, the proposed prognostic function can be

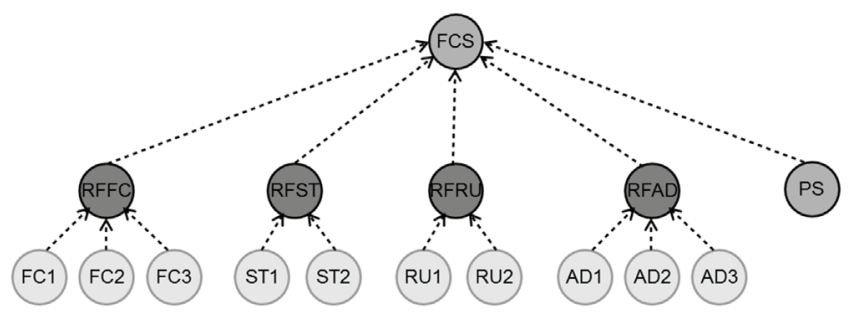

Fig. 5. Model of flight control service with redundant components. considered as a service as it is defined in Service Oriented Architecture (SOA) that lead to distributed enterprises applications [41]. This service is solicited by production management wanting to plan productive tasks and it provides decision supports indicators used jointly by production and maintenance management to validate or to modify the production tasks scheduling and to plan maintenance interventions. Therefore, OOBNs are sufficient tools to implement it.

The computing rules use the model of the system and the local prognostics to infer the decision support indicators for joint production and maintenance planning. Those rules are methods implemented for the different of classes of nodes.

\section{Inference rules}

The aim of the computing rules is to provide decision support indicators for maintenance and production planning from the fusion of local prognostics and the model of the complex system. These decision support indicators shall help the maintenance and production planning to make decision according to criteria such as risk, cost, reliability and availability. Risk is often considered as the combination of vulnerability and criticality, which can also be considered as a combination of probability and impact. Usually, impacts can be costs, casualties, damages to environment... Knowing the impacts, probability or reliability criteria are more appropriate for decision supports regarding the assignment of productive tasks to a system or regarding its maintenance [36]. In this case, a prognostic should be considered as the probability of failure or of survival in a given duration of use which includes planned productive tasks rather than as the remaining duration of use associated with a confidence or imprecision indicator $[20,29]$. That is why studies aim at defining degradation prediction models that provide probability density functions of the duration of use knowing the maximum degradation level like in $[19,49,9]$ or of the degradation level knowing the duration use like in [14]. These models may require the expected uses of the systems that may be introduced thanks to parameters representing the severity of the future tasks [10]. The expected uses, and thus their severities, can be anticipated by production planning that assigns tasks to systems. Thus the definition of always more accurate degradation prediction models is also one of the main issues in the domain of prognostics [12]. Indeed, according to CBM, components and even structural parts must be maintained before they reach one of their maximum degradation levels. Therefore we assume the local prognostics, which run models predicting the evolvements of the degradations for the planned tasks or predicting the RULs of the components, provide the PDFs or the Cumulative Probability Distribution Functions (CPDFs) of those predicted evolvements or of those RULs.

Prognostic functions cannot be implemented for all the components of a complex system for cost or space reasons. Thus a complex system is not completely observed in terms of prognostics. Nevertheless, component suppliers can define the PDFs or CPDFs of failure of components depending on their uses (duration or number of cycles) thanks to statistical studies. These studies may correspond to those aiming at defining the probabilities of elementary failures [11], the Mean Time To Failure (MTTF) of the components. Knowing the PDF or the CPDF of the TTF or the TBF of a component based on its passed use and on its expected use, the probability of its failure before the fulfilment of the planned tasks can be calculated [44]. We therefore assume that we have the PDFs or the CPDFs of the TTFs or the TBFs of the components or even structure elements without monitoring. The probabilities of their failures before the fulfilment of the planned tasks, which are determined from their expected uses, are considered as local prognostics. 

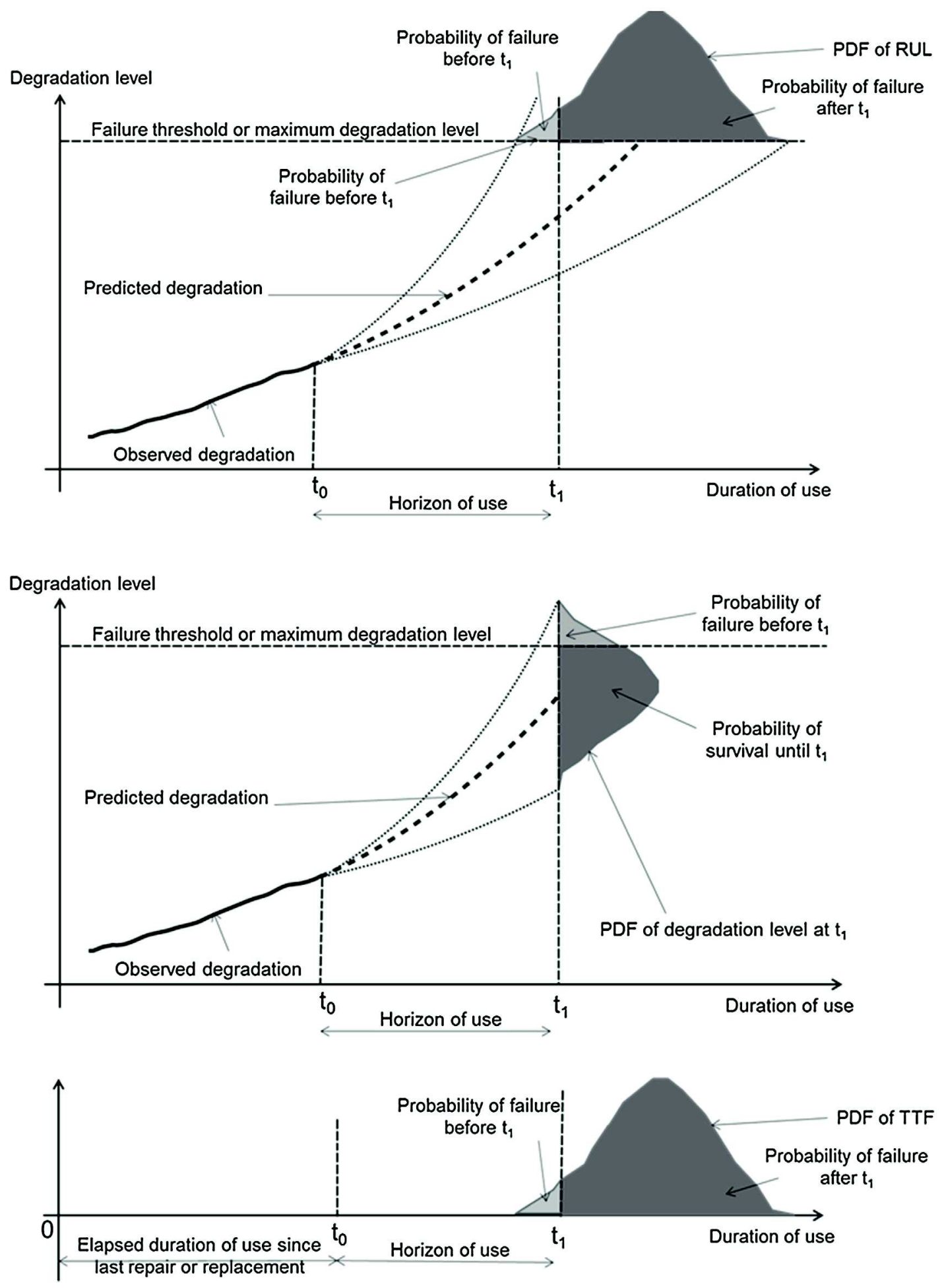

Fig. 6. PDFs of the predictions of RULs, of the evolvement of degradations and of the TTFs.

The Fig. 6 illustrates PDFs of the predictions of RULs, of the evolvement of degradations and of the TTFs where $t_{0}$ is the current date and $t_{1}-t_{0}$ the planned duration of use which can also be called the horizon of use. Let us note the PDFs and CPDFs can be given relatively to number of cycles and not relatively to the duration of use. In such cases, the number of cycles must be determined for all the planned tasks.

Once the probabilities of failures before the end of the planned tasks are determined from the local prognostics, the proposed generic function compute the probabilities the components, the functions, the subsystems and the system are not able to fulfill the planned tasks according to the complex system model. These probabilities are the first decision support indicators for making decision like to validate the planned tasks, to reduce the number of the planned tasks before maintenance, to maintain the system . . . Therefore, the graph that models a complex system, as described in Section 2, is made of nodes that contain the probabilities the components, the functions, the subsystems and even the complex 
system fail in fulfilling the planned productive tasks if they solicit all its functions. The proposed generic function computes, for each node, the probabilities to switch into undesired states before the fulfillment of these tasks. This computation is done thanks to OOBN ability to compute the probabilities of random variables knowing their conditional dependencies [21].

However, the knowledge of those probabilities of the disabilities to fulfill of the scheduled tasks is not sufficient regarding CBM policies and "intelligent prognostics" as defined in [24]. Indeed, maintenance need to know the components that need to be fixed or replaced to reduce the probability that the system fails to complete its assigned tasks in order to shorten downtimes by planning in advance the maintenance actions and their logistics. That is why the proposed generic function also provide decision support indicators about the components and structure elements whose maintenance will most increase the abilities of the components, functions, subsystems and system to fulfill the planned tasks. Defining these indicators is the object of the extension we propose to OOBN. Thus decision to maintain or not to maintain components can be made according to the probabilities of failure of the entities (components, functions, subsystems ... ), to the operational risk assessment, to the costs of maintenance operations ... $[9,10,20,30]$. In the rest of the paper, structure elements are considered as components.

\subsection{For components}

According to CBM policies, maintenance is done before the failure and is conditioned to the health status of the component. So we can here consider that the failure of a component will not cause any damage to other components because the failure is not supposed to happen thanks to preventive maintenance which can be planned according to the results the proposed prognostic function. In this context, the probabilities of transition between five states can be considered for each component. These states are:

- OK: The component is able to operate within its minimum performances required to fulfill the planned tasks even if its performances are not the best ones because of incipient degradations or of a little more important degradations.

- F: The component is failed. This means that the component is not able to operate within its minimum performances required to fulfill the planned tasks. This state is for failures that have internal origins. The component will not recover its specified performances if it does not undergo maintenance.

- 00: The component is out of order. This means that the component is not able to operate within the minimum performances required to fulfill the planed tasks but without requiring maintenance. This state corresponds to failures whose origins are not internal but it is the consequence of the inability of at least one other entity to provide a function or a service the component needs to operate. To recover its performances required to fulfill the planned tasks, the maintenance of the component itself is useless but one or more other components have to undergo maintenance.

- FOO: This state is a combination of the F state and the 00 state.

- KO: This state means that the component is not able to operate within its minimum performances required to fulfill the planned tasks because its state is F, OO or FOO. KO stands for "not OK".

The graph of Fig. 7 shows the Markov model of a component states where $\lambda_{F}$ is the probability that the component fails because of at least one failure with internal origin, $\lambda_{00}$ is the probability that the component fails because of external reasons, $\mu_{F}$ is the

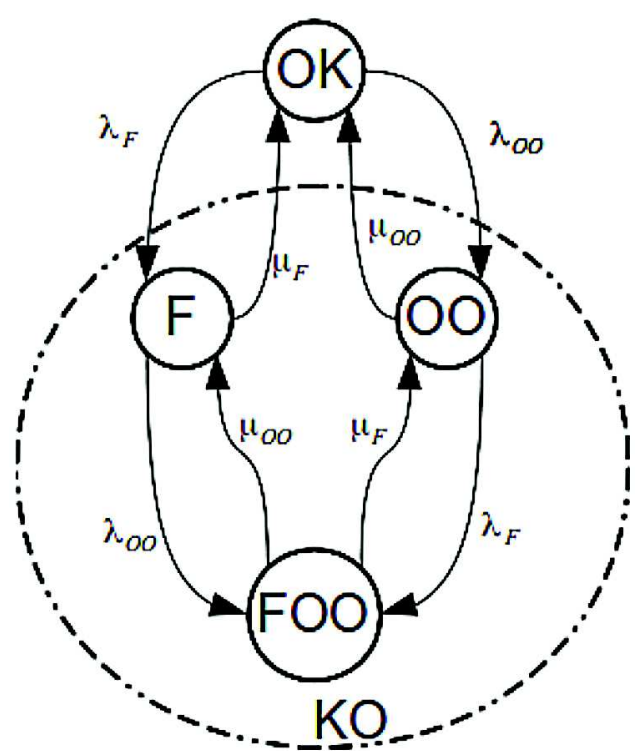

Fig. 7. Graph of transitions between the considered states of a component.

probability that the component recovers from the F state and $\mu_{0 O}$ is the probability that the component recovers from the F state.

Here, the considered components do not have any self-healing abilities. Therefore maintenance is required to bring a component back in OK state. As one of the aim of the prognostic function is to define if components need maintenance, the $\mu_{F}$ and $\mu_{O O}$ transitions are not considered. Once a component is in the F state or in the $\mathrm{OO}$ state, it is no longer able to operate within its minimum performances required to fulfill the planned tasks without maintenance. So, it is in KO state. The consequences of KO states of components must be assessed to define the ability of the complex system to perform the future tasks. Nevertheless, it is interesting to distinguish the $\mathrm{F}$ state from the $\mathrm{OO}$ state because it makes it possible to guide maintenance intervention to the component itself or to other components.

In order to provide information about the ability of the complex system to perform the future tasks and, also, information about components that need maintenance, four fields (named attributes in object oriented approach) will be computed for each node that belongs to a component class:

- $\lambda_{F}^{C x}(t)$ : the probability that the component $C x$ is in the F state before instant $t$,

- $\lambda_{O O}^{C x}(t)$ : the probability that the component $\mathrm{Cx}$ is in the $\mathrm{OO}$ state before instant $t$,

- $\lambda_{K O}^{C X}(t)$ : the probability that the component $C x$ is in the KO state before instant $t$; this probability is the inverse probability that the component $C x$ is still in the OK state until instant $t \lambda_{O K}^{C x}(t)$ and so:

$\lambda_{K O}^{C X}(t)=1-\lambda_{O K}^{C X}(t)$

- $i d^{C x}(t)$ : the identifier of the component that should firstly undergo maintenance to reduce $\lambda_{K O}^{C x}(t)$; it may be the component's own identifier.

Knowing the proposed generic function provides decision support indicators for maintenance and production planning, $t$ is the time at which the planned tasks will be completed. 
$\lambda_{F}^{C x}(t)$ is computed from the set local prognostics $\boldsymbol{P}^{x}=$ $\left\{P_{i}^{\chi}(t) \mid i=1, \ldots, n\right\}$ of the component Cx where $n$ is the number of failure modes of $C x$. These local prognostics are defined either thanks to prognostic functions that use the observed degradations, the degradation model and the expected uses of the component or thanks to statistical data dealing, for example, with the TTFs of the component based on its passed uses and on its expected uses. A local prognostic $P_{i}^{X}(t)$ is the probability that the failure mode $i$ of the component $C x$ occurs before $t$. Therefore $\lambda_{F}^{C x}(t)$ consists of the probability that, at least, one failure mode of $\mathrm{Cx}$ will occur before $t$ or of the inverse probability that none of the failure modes will occur before $t$.

$\lambda_{F}^{C x}(t)=1-\prod_{i=1}^{n}\left(1-P_{i}^{x}(t)\right)$

The $\lambda_{O O}^{C x}(t)$ computation requires to know the set of the predecessors of $\mathrm{Cx}$ in the modeling graph $\boldsymbol{G}$. These predecessors are entities (components or functions) noted Ei. This set is $\Gamma^{-1}(C x)=$ $\{E i \mid(E i, C x) \in \boldsymbol{A}\}$ where $\boldsymbol{A}$ is the set of the arcs of the graph $\boldsymbol{G}$. CX will be out of order before $t$ if, at least, one $E i \in \Gamma^{-1}(C x)$ will become KO before $t$. Thus $\lambda_{O O}^{C x}(t)$ is the inverse probability that none of the $E i \in \Gamma^{-1}(C x)$ will become KO before $t$.

$\lambda_{O O}^{C x}(t)=\left\{1-\prod_{E i \in \Gamma^{-1}(C x)}\left(1-\lambda_{K O}^{E i}(t)\right), \quad \Gamma^{-1}(C x) \neq \emptyset\right.$

$\lambda_{K O}^{C X}(t)$ can so be considered as the inverse probability that neither $\mathrm{Cx}$ will fail nor $\mathrm{Cx}$ will become out of order before $t$.

$\lambda_{K O}^{C X}(t)=1-\left(1-\lambda_{F}^{C X}(t)\right)\left(1-\lambda_{O O}^{C X}(t)\right)$

$i d^{C x}(t)$ is defined assuming that if a component Cy has just been maintained then $\lambda_{F}^{C y}(t)=0$. This assumption is also correct if each component $\mathrm{Cx}$ of the complex system has its attribute $\lambda_{F}^{C x}(t)$ reset to the same very low value (e.g. 1e-10) after they had been maintained. In that case, the component $\mathrm{Cy}$ that should firstly undergo maintenance in order to reduce at most the value of $\lambda_{K O}^{C x}(t)$ is such as:

$\left.\lambda_{F}^{C y}(t)=\max \lambda_{F}^{C x}(t), \max _{E k \in A^{-1}(C x)}\left(\lambda_{F}^{E k}(t)\right)\right)$

where $A^{-1}(C x)$ is the set of the nodes from which the node $C \mathrm{x}$ is attainable and Ek is a node that can be a component, a simple function or a redundancy function. An entity Ek belongs to $A^{-1}(C x)$ if it exists at least one path from Ek to $C x$ in the directed graph $\boldsymbol{G}$. The rule (5) is demonstrated in the Appendix A.

From an implementation point of view, $i d^{C x}(t)$ can be defined by the following recursive rule: "Whether the $\lambda_{F}^{C x}(t)$ of the component is the main contributor to $\lambda_{K O}^{C x}(t)$, and so $i d^{C x}(t)$ receives the identifier $C_{X}$, or the $\lambda_{F}^{E k}(t)$ of one of its predecessors is the main contributor to $\lambda_{K O}^{C x}(t)$. If it is the $\lambda_{F}^{E k}(t)$ of one of its predecessor, the $\lambda_{F}^{E k}(t)$ of this predecessor Ek is whether the main contributor to $\lambda_{K O}^{E k}(t)$, and so $i d^{C X}(t)$ receives the identifier Ek, or the $\lambda_{F}^{E l}(t)$ of one of its predecessors is the main contributor to $\lambda_{K O}^{E k}(t)$. If it is ...". To avoid to look for all the entities of $A^{-1}(C x)$ and thus to reduce the complexity of the algorithm, the attribute $\lambda_{F_{\text {max }}}^{E k}(t)$ is introduced and (5) can be implemented by the following algorithm:

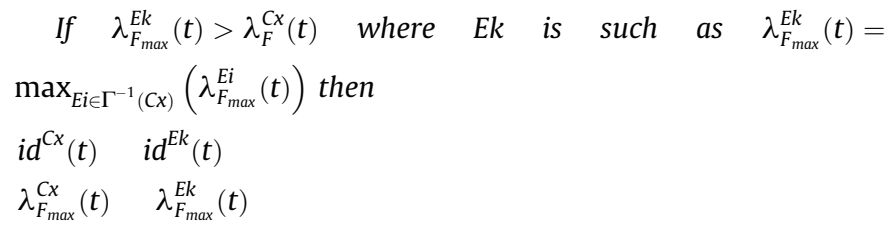

else

$i d^{C x}(t) \quad C x$

$\lambda_{F_{\max }}^{C x}(t) \quad \lambda_{F}^{C x}(t)$

end ifwhere, the attribute $\lambda_{F_{\max }}^{C x}(t)$ contains the value of determined from (5). This attribute enables to propagate recursively the value that maximizes $\lambda_{K O}^{C z}(t)$ of a component $\mathrm{Cz}$.

This rule may not be optimal regarding the minimization of $\lambda_{K O}^{C x}(t)$, if the values $\lambda_{F}(t)$ of components are not reset to a same value after they had been maintained whatever the components are. The rule (5) and its implementation (6) consider neither maintenance costs nor notions of risk but just occurrence probabilities. Here, the aim is to implement a rule to show how the prognostic function can provide a decision support indicating the entity that should first be maintained. So this rule can be adapted to maintenance policies of the company that exploits the system or to the structure of maintenance costs for the different components as proposed in [30].

\subsection{For simple functions}

Functions are implemented by the means of components and sub-functions. Simple functions will fail or become out of order before $t$, the time at which the planned task will be completed, if at least one of their entities fails or becomes out of order before $t$. Considering that an entity, which belongs to a simple function, is maintained before its failure according to CBM preventive policies and thanks to the indicators provided by the proposed function, the failure of this entity will not damage of other entities because it is not supposed to happen. Thus, two states can be considered for each simple function:

- OK: The simple function is able to operate within its minimum performances required to fulfill the planned tasks.

- OO: The simple function is out of order. This means that it is not able to operate within its minimum performances required to fulfill the planned tasks because of the failure of, at least, one of its entities or because, at least, one of its entities is out of order. This state also corresponds to the KO state.

Therefore the probability $\lambda_{K O}^{S F x}(t)$ of a simple function SFx to become KO before $t$ can be related to its probability $\lambda_{0 O}^{S F x}(t)$ to become out of order and to its probability $\lambda_{O K}^{S F x}(t)$ of survival until $t$ thanks to:

$\lambda_{K O}^{S F x}(t)=\lambda_{O O}^{S F x}(t)=1-\lambda_{O K}^{S F x}(t)$

Thus only two attributes will be computed for each node that belongs to the simple function class:

- $\lambda_{K O}^{S F x}(t)$ : the probability that the simple function SFx will be in the KO state before $t$,

- $i d^{S F x}(t)$ : the identifier of the component that should firstly undergo maintenance to reduce $\lambda_{K O}^{S F x}(t)$. 
$\lambda_{K O}^{S F x}(t)$ is computed from the set of the predecessors of SFx in the modeling graph $\boldsymbol{G}$. These predecessors are entities (components, simple functions or redundancy functions) noted Ei. This set is $\Gamma^{-1}(S F x)=\{E i \mid(E i, S F x) \in \boldsymbol{A}\}$. SFx will be out of order before $t$ if, at least, one $E i \in \Gamma^{-1}(S F x)$ becomes KO before $t$. Thus $\lambda_{K O}^{S F x}(t)$ and $\lambda_{O O}^{S F x}(t)$ are the inverse probabilities that none of the $E i \in \Gamma^{-1}(S F x)$ will become KO before $t$.

$\lambda_{K O}^{S F x}(t)=\lambda_{O O}^{S F x}(t)=1-\prod_{E i \in \Gamma^{-1}(S F x)}\left(1-\lambda_{K O}^{E i}(t)\right)$

The rule that enables to determine $i d^{S F x}(t)$ is derived from the rule (5) knowing that the simple function cannot be the main contributor to $\lambda_{K O}^{S F x}(t)$ because it does not have $\lambda_{F}^{S F x}(t)$ attribute that also corresponds to $\lambda_{F}^{S F x}(t)=0$. Thus the component Cy that should firstly undergo maintenance in order to decrease at most the value of $\lambda_{K O}^{S F x}(t)$ is such as:

$\lambda_{F}^{C y}(t)=\max _{E k \in A^{-1}(S F x)}\left(\lambda_{F}^{E k}(t)\right)$

Where an entity Ek belongs to $A^{-1}(S F x)$ the set of the nodes from which the node SFx is attainable if it exists, at least, one path from Ek to SFx in the directed graph $\boldsymbol{G}$.

From an implementation point of view, $i d^{S F x}(t)$ can be determined by the following algorithm that is derived from (6) knowing that $\lambda_{F}^{S F x}(t)=0$ :

$$
\begin{array}{ll}
i d^{S F x}(t) & i d^{E k}(t) \\
\lambda_{F_{\max }}^{S F x}(t) & \lambda_{F_{\max }}^{E k}(t)
\end{array}
$$

where Ek is such as $\lambda_{F_{\text {max }}}^{E k}(t)=\max _{E i \in \Gamma^{-1}(S F x)}\left(\lambda_{F_{\text {max }}}^{E i}(t)\right)$ where, the attribute $\lambda_{F_{\text {max }}}^{S F x}(t)$ contains the value determined from (9).

\subsection{For redundancy functions}

A redundancy function ensures its service until all the redundant entities are failed or become out of order. But if there is only one entity that is able to perform the service, there is no more redundancy and, in many cases, the system must not begin a new task mainly because of safety reasons [24]. Therefore three states can be considered for each redundancy function:

- OK: The redundancy function is able to operate within its minimum performances its minimum performances required to fulfill the planned tasks thanks to one of its entities at least.

- 0O: The redundancy function is out of order. This state means that the redundancy function is not able to operate within its minimum performances its minimum performances required to fulfill the planned tasks and thus this state also corresponds to the KO state. This state also means that none of the entities of the redundancy function is able to provide the service they implement within the minimum performances its minimum performances required to fulfill the planned tasks.

- LR: The redundancy is lost. This state means that only one entity that implements the redundancy function is able to provide the service within its minimum performances its minimum performances required to fulfill the planned tasks. When a redundancy function is in the LR state, the complex system should not begin a new task mainly because of safety reason.

Therefore for each node that belongs to the redundancy function class, three attributes will be computed for $t$, the time at which the planned tasks will be completed:
- $\lambda_{K O}^{R F x}(t)=\lambda_{O O}^{R F x}(t)$ : the probability that the redundancy function RFx will be in the KO state before $t$,

- $\lambda_{L R}^{R F x}(t)$ : the probability that the redundancy function RFx will be in the LR state before $t$,

- $i d^{R F x}(t)$ : the identifier of the component that should firstly undergo maintenance to reduce $\lambda_{K O}^{R F x}(t)$ and $\lambda_{L R}^{R F x}(t)$.

$\lambda_{K O}^{R F x}(t)$ is computed from the set of the predecessors of RFx in the modeling. These predecessors are entities (components, simple functions or redundancy functions) noted Ei. This set is $\Gamma^{-1}(R F x)=\{E i \mid(E i, R F x) \in \boldsymbol{A}\}$. RFx will fail before $t$ if all the entities $E i \in \Gamma^{-1}(R F x)$ will fail before $t$. Therefore:

$\lambda_{K O}^{R F x}(t)=\lambda_{O O}^{R F x}(t)=\prod_{E i \in \Gamma^{-1}(R F x)} \lambda_{K O}^{E i}(t)$

However, redundancy will be lost before $t$ if less than two entities $E i \in \Gamma^{-1}(R F x)$ will survive until $t$. Therefore:

$$
\begin{aligned}
\lambda_{L R}^{R F x}(t) & =\lambda_{K O}^{R F x}(t) \\
& +\sum_{E i \in \Gamma^{-1}(R F x)}\left\{\left(1-\lambda_{K O}^{E i}(t)\right) . \prod_{E j \in \Gamma^{-1}(R F x), j \neq i} \lambda_{K O}^{E j}(t)\right\}
\end{aligned}
$$

The quantity, if it was zero, would decrease at most $\lambda_{K O}^{R F x}(t)$ and $\lambda_{L R}^{R F x}(t)$ is $\lambda_{K O}^{E k}(t)=\max _{E i \in \Gamma^{-1}(R F x)}\left(\lambda_{K O}^{E i}(t)\right)$. However, the maintenance of a component Cy only reduces its $\lambda_{F}^{C y}(t)$. Thus, for a redundant function that is only composed of components without any predecessor, the component that should firstly undergo maintenance is the component $\mathrm{Ck}$ that verifies $\lambda_{F}^{C k}(t)=\max _{C i \in \Gamma^{-1}(R F x)}\left(\lambda_{F}^{C i}(t)\right)$. For a redundancy function that is composed of components without predecessor and also of components with predecessors and/or functions, the maximization of $\lambda_{K O}^{R F x}(t)$ will lead to favor the maintenance of components without predecessor. This is not really relevant regarding to the CBM policies whose one aim is to maintain components just before their failures. That is why we here choose the rule adapted from (9) for which the component $\mathrm{Cy}$, which should firstly undergo maintenance, is the one that verifies $\lambda_{F}^{C y}(t)=\max _{C x \in A^{-1}(R F x)}\left(\lambda_{F}^{C x}(t)\right)$ where $A^{-1}(R F x)$ is the set of the nodes from which the node RFx is attainable. This rule is not optimal for every cases regarding the decreasing of $\lambda_{K O}^{R F x}(t)$ and $\lambda_{L R}^{R F x}(t)$ values if there are functions or components with predecessors in RFx; but it is compliant to the CBM policies.

Because of the relationship (11), the rules (5) and (9) and their respective implementations (6) and (10) do not seem to be relevant when a redundant function RFx belongs to the predecessors of a simple function or of a component Ek. However if the $\lambda_{K O}^{R F x}(t)$ of the redundant function is compared to the $\lambda_{F_{\text {max }}}^{S F x}(t)=\max _{E l \in A^{-1}(E k)}\left(\lambda_{F}^{E l}(t)\right)$ for a simple function SFx or to the $\lambda_{F_{\text {max }}}^{C_{x}}$ determined from (5) for a component $\mathrm{CX}$ and if $\lambda_{K O}^{R F x}(t)>\lambda_{F_{\max }}^{S F x}$ or $\lambda_{K O}^{R F x}(t)>\lambda_{F_{\max }}^{C x}$, one can consider that $\lambda_{K O}^{R F x}(t)$ must be reduced in order to decrease $\lambda_{K O}^{E k}(t)$ with $\mathrm{Ek}=\mathrm{SFx}$ or Ek $=\mathrm{Cx}$. However this should not happen. Indeed, the components must be maintained before $\lambda_{L R}^{R F x}(t)$ reaches a critical threshold in order to keep a minimal safety level and according to (12) $\lambda_{L R}^{R F x}(t)>\lambda_{K O}^{R F x}(t)$. Consequently, the respective implementations of rules (5) and (9) must be modified in such a way the algorithm (6) thus becomes:

If $\lambda_{F_{\text {max }}}^{E k}(t)>\lambda_{F}^{C x}(t)$ 
where $E k$ is such as $\lambda_{F_{\text {max }}}^{E k}(t)=\max _{E i \in \Gamma^{-1}(C x)}\left\{\max _{E i \notin F_{R}}\left(\lambda_{F_{\max }}^{E i}(t)\right)\right.$, $\left.\max _{E i \in F_{R}}\left(\lambda_{K O}^{E i}(t)\right)\right\}$ then

$$
\begin{array}{lc}
i d^{C x}(t) & i d^{E k}(t) \\
\lambda_{F_{\text {max }}}^{C x}(t) & \lambda_{F_{\text {max }}}^{E k}(t)
\end{array}
$$

else

$i d^{C x}(t) \quad C x$

$\lambda_{F_{\max }}^{C x}(t) \quad \lambda_{F}^{C x}(t)$

end ifwhere $F_{R}$ is the set of nodes that are redundancy functions of the complex system. The algorithm (13) is applied for a component $\mathrm{Cx}$.

And the algorithm (10) becomes:

$$
\begin{array}{lc}
i d^{F x}(t) & i d^{E k}(t) \\
\lambda_{F_{\text {max }}}^{F x}(t) & \lambda_{F_{\text {max }}^{E k}}^{E k}(t)
\end{array}
$$

where $E k$ is such as $\lambda_{F_{\text {max }}}^{E k}(t)=\max _{E i \in \Gamma^{-1}(F x)}\left\{\max _{E i \notin F_{R}}\left(\lambda_{F_{\text {max }}}^{E i}(t)\right)\right.$, $\left.\max _{E i \in F_{R}}\left(\lambda_{K O}^{E i}(t)\right)\right\}$ where $F_{R}$ the set of redundancy functions of the complex system. The algorithm (14) is applied whether for a simple function or a redundancy function Fx.

\subsection{Algorithm of the generic function}

The generic function providing the decision support indicators consists of a graph traversal in which the attributes of the nodes are computed from the attributes of the nodes that belongs to the sets of their predecessors. This function consists of five main steps:

1. The modeling graph of the complex system is instantiated.

2. The attributes $\lambda_{K O}, \lambda_{F}, \lambda_{O O}, \lambda_{L R}$ and $\lambda_{F \max }$ of the nodes are set to zero and the attributes id are set to a default value. This default value means that the prognostic function has not been processed.

3. The local prognoses are computed from their current ages and conditions [36], and the future operations profiles that are transmitted by production planning that assigns tasks to systems, especially if the local prognostic functions are model based [11]. Knowing how the future productive tasks should solicit the functions and subsystems of the system, acceptable levels of degradation could also be adjusted for the computation of the local prognostics under which the productive tasks would be fulfilled.

4. For each local prognostic, the attributes $\lambda_{F}, \lambda_{O O}, \lambda_{K O}$, id and $\lambda_{F \max }$ of the involved node of component type are calculated from (2), (3), (4) and (13).

4.1. If the attributes $\lambda_{K O}$, id or $\lambda_{\text {Fmax }}$ of the node have been modified, for each successor of the node, the attributes $\lambda_{F}, \lambda_{O o}$, $\lambda_{L R}, \lambda_{K O}, \lambda_{F \max }$ and $i d$ are calculated from (2), (3), (4), (8), (11), (12), (13) and (14) according to the type of the node.

4.2. Then 4.1 .

5. Then, for each node and according to its type, the attributes $\lambda_{F}$, $\lambda_{O O}, \lambda_{K O}, \lambda_{L R}$ and $i d$ are displayed or sent to provide decision support indicators for production and maintenance planning.

The proposed generic function can also be used when a new local prognosis is provided. In that case, the function begins at the step 4 which becomes "for the new local prognosis, the attributes $\lambda_{F} \lambda_{O O}, \lambda_{K O}$, id and $\lambda_{F \max }$ of the involved component are calculated from (2), (3), (4) and (13)".
In order to validate the proposed prognostic function and the system modeling, the bridge system and also the Kamat-Riley system proposed in [38] have been modeled and computed by the proposed prognostics function. The results the prognostic function has produced have been compared to the one obtained with Netica software. The results produced by prognostic function and Netica software were the same. For those comparisons, components have had only one local prognostic each, redundancy functions were considered as "or" gates and simple functions as "and" gates. However, computations made by Netica have not produced the intermediate results for the "or" gates and the "and" gates that the proposed prognostic function can produce for each attribute of each node.

The modeling of other systems may require transformations to be computed by the proposed function based on OOBN.

\subsection{Modeling graph transformations}

As implied by the step 4, the proposed generic function for fusing the local prognoses is a recursive graph traversal. Therefore, if the graph modeling the complex system has graph cycles, the algorithm made of the steps 1 to 5 will never end. As the modeling of complex systems and the proposed generic function are based on Bayesian networks that are acyclic graphs [21], graph cycles of the complex system model must be suppressed. Therefore, the graph cycles must be identified as this is illustrated through the example presented in Fig. 8(a) where the hatched area corresponds to a graph cycle. This identification can be automatically done by the Tarjan's algorithm [42]. Those cycles may appear while modeling the system from design models such as SADT diagrams or diagrams proposed in SysML like activity diagrams or internal block diagrams. In those design models an entity A can produce a flow $f a$ that is the input of an entity $B$ that produce a flow $f b$ that is also used by the entity $A$. A direct consequence is that if $A$ does not produce $f a$ B will not produce $f b$ and if $B$ does not produce $f b$ A will

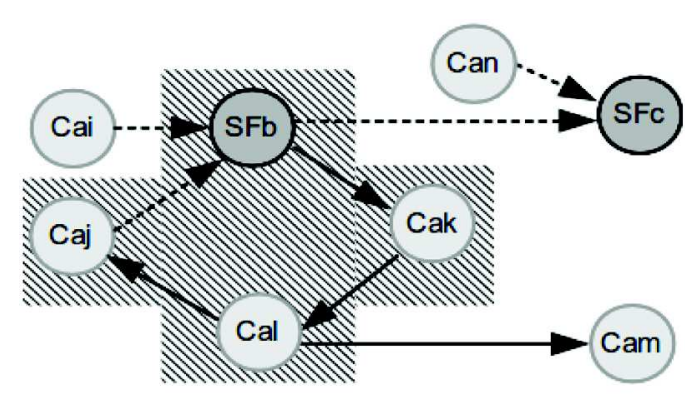

(a)

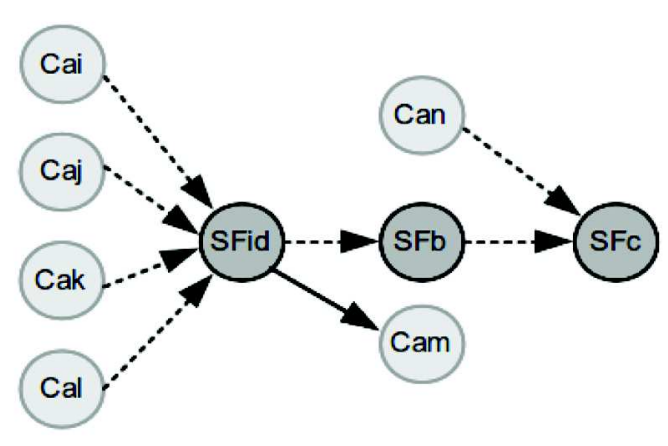

(b)

Fig. 8. Transformation of a graph cycle (a) into a simple function of interdependence (b). 
not produce the flow fa. Thus, if one of both the entities cannot operate, the second one cannot operate too. Considering that $A$ and $B$ implement a simple function, this function will be able to operate only if A and B are both able to operate. But cycles may involve more than two entities that can be functions and components. However, redundancy functions should not be involved in those patterns. Indeed, redundant entities or sets of entities are mainly designed because of safety reasons and that is why these entities, which ensure the same function, are very independent as mentioned in [24]. In the cases of graph cycles, like the one described in Fig. 8(a), the entities cannot operate normally if at least one entity in the cycle is failed or out of order. A graph cycle can be transformed by introducing a simple function as suggested by the example with two entities. We here call these introduced simple functions "functions of interdependence". This transformation leads to replace causal relationships by membership relations and vice versa. However, arcs of the complex system modeling graph are unweighted and they just direct the graph traversals. So, this replacement does not impact the computation of the different attributes of the nodes. Three steps lead to transformation of the complex system modeling graph:

1. The simple function of interdependence gathers all the components included in the cycle as well as the components of simple functions included in the cycle.

2. The simple function of interdependence becomes the unique entity of each function that contains at least one component that belongs to a simple function included in the cycle.

3. The components that were the tails of causal relationships with components that now belong to the simple function of interdependence are then connected to the simple function of interdependence, which is the head of causal arcs, for which those components are still the tails.

The example shown in Fig. 8 illustrates those modeling graph transformations where SFid is the introduced simple function of interdependence.
Once graph cycles of the modeling graph of the complex system are suppressed, several paths may exist from a given node to another node. Those several paths can be the consequence of the design models such as SADT diagrams, activity diagrams or internal block diagrams. In the proposed modeling, the existence of several paths from a node E1 to a node E2 will introduce several times the value of $\lambda_{K O}^{E 1}(t)$ into the computations of $\lambda_{K O}^{E 2}(t)$ and $\lambda_{O O}^{E 2}(t)$, according to the proposed given recursive algorithm and the relationships (3), (8), (11) and (12) it implements, whereas $\lambda_{K O}^{E 1}(t)$ is the probability of a unique event that must so be considered only once.

To avoid this, the computation of the attributes of the nodes of the modeling acyclic graph of the complex system can be done by generating several graphs from the modeling graph such as they have only one path from one node to another one. The graph that is obtained from downstream to upstream of the modeling acyclic graph until there are at least two paths between a downstream node Ed and an upstream node Eu. In this first graph, the attributes of Ed will not be computed by the proposed function. The node Ed and the part of the graph, which is downstream, will be computed thanks to a second graph. In this second graph, the relationships between the node Eu and the nodes from which Ed can be reached are suppressed and Eu is directly connected to Ed. The computation of the attributes of the nodes of this new graph are then computed but only the attributes of the nodes that have not been computed for the first graph are stored or displayed. This new computation is done until there are, in this new graph, at least two paths between two nodes. A third graph must then be built according to the same procedure and so on. Nevertheless there is an exception if an upstream node Ed belongs to a redundancy function RFx, the path that goes through the redundancy function is not considered as a second path. Indeed in this case, $\lambda_{K O}^{E d}(t)$ decreases $\lambda_{K O}^{R F x}(t)$ from (11) but increases $\lambda_{K O}^{E u}(t)$ of any downstream nodes Exy that can be reached by paths that do not have redundancy function from (4) and (8). Indeed, a redundancy function can be assimilated as "or" gates whereas simple functions and components can be

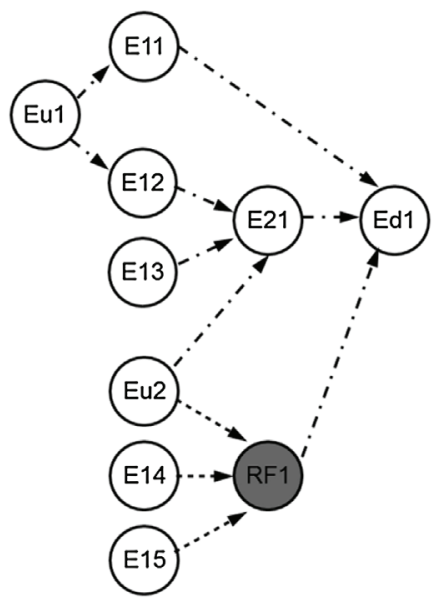

(a)

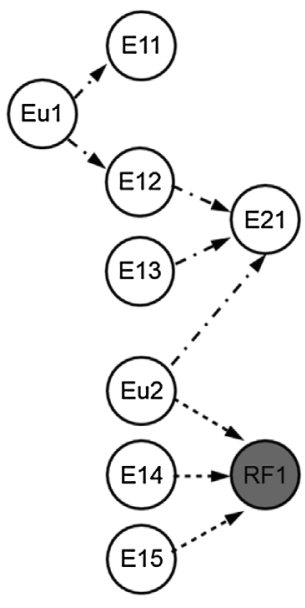

(b)

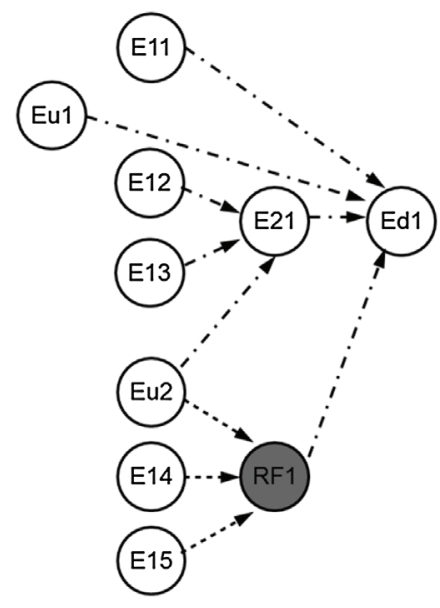

(c)

-.-Causal or membership relation

Membership relation

Exy Simple function or component

Redundancy function

Fig. 9. Example of graphs generated to avoid to introduce several times the probability of the KO state of one node. 
assimilated as "and" gates in the modeling based proposed by Simon et al. for the reliability of complex systems [38]. However, the generic function we propose computes probabilities for those functions.

To illustrate the procedure to generate the graphs, an example is presented in Fig. 9. In Fig. 9, the graph (a) is the one obtained after the transformations to suppress graph cycles. In this graph there are two paths between the nodes Eu1 and Ed1. There are also two paths between the nodes Eu2 and Ed1. The graph (b) is obtained from (a) by suppressing the node Ed1. The proposed generic function is then computed for the graph (b) and the values obtained for the attributes of the nodes of (b) are stored. The next step consists in building a graph to compute the attributes of Ed1 which is the graph (c). In this graph, Eu1 is directly connected to Ed1 such as $\lambda_{K O}^{E u 1}(t)$ is introduced only once in the computation of $\lambda_{K O}^{E d 1}(t)$. The generic function is then computed for the graph (c) but only the values of the attributes of Ed1 are stored. However, there are two paths between Eu2 and Ed1 in graph (c) but Eu2 belongs the redundancy function RF1 which corresponds to the exception described previously.

\subsection{Using the attributes computed for each nodes}

For production planning, the stored attributes $\lambda_{K O}$ and $\lambda_{L R}$ are indicators that assess the probability that the functions required by the planned tasks will fail while fulfilling them. According to the values of these indicators, production planning may choose to validate the assignment of productive tasks, to reduce the number of assigned tasks, to change the assigned tasks and/or to schedule downtimes for maintenance.

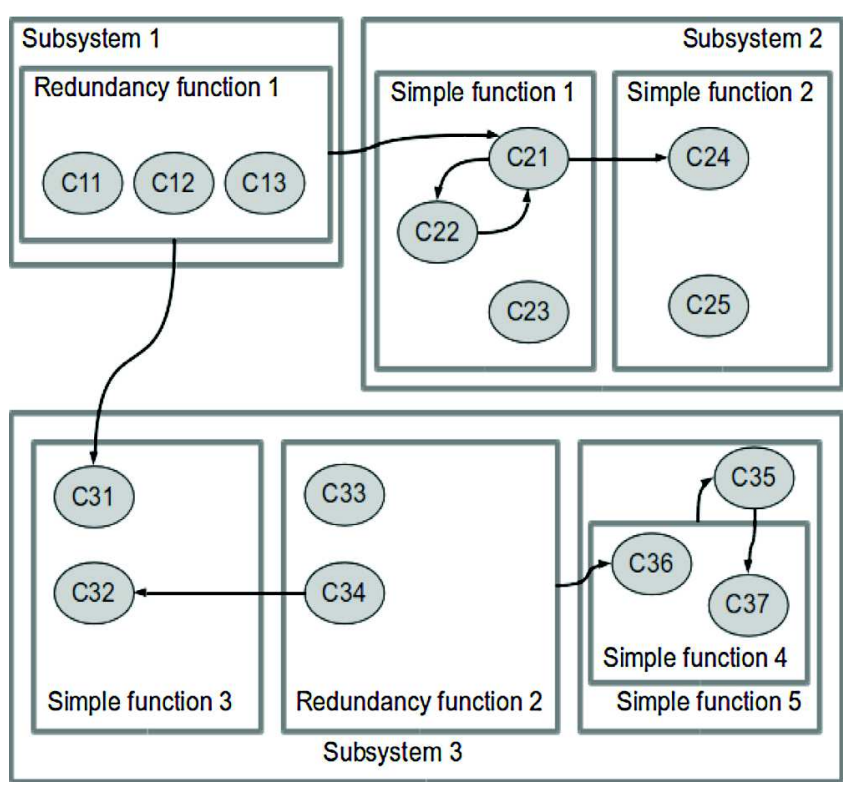

Fig. 10. Fictitious multi-component system.

For maintenance planning, the stored attributes $\lambda_{K O}, \lambda_{F}, \lambda_{O O}, \lambda_{L R}$ and/or $i d$ are indicators that aim at helping maintenance to choose the components that should be maintained. The attribute id indicates the component that shall first undergo maintenance to better increase the probability that the entity (function or component) survives until the completion of the planned tasks.

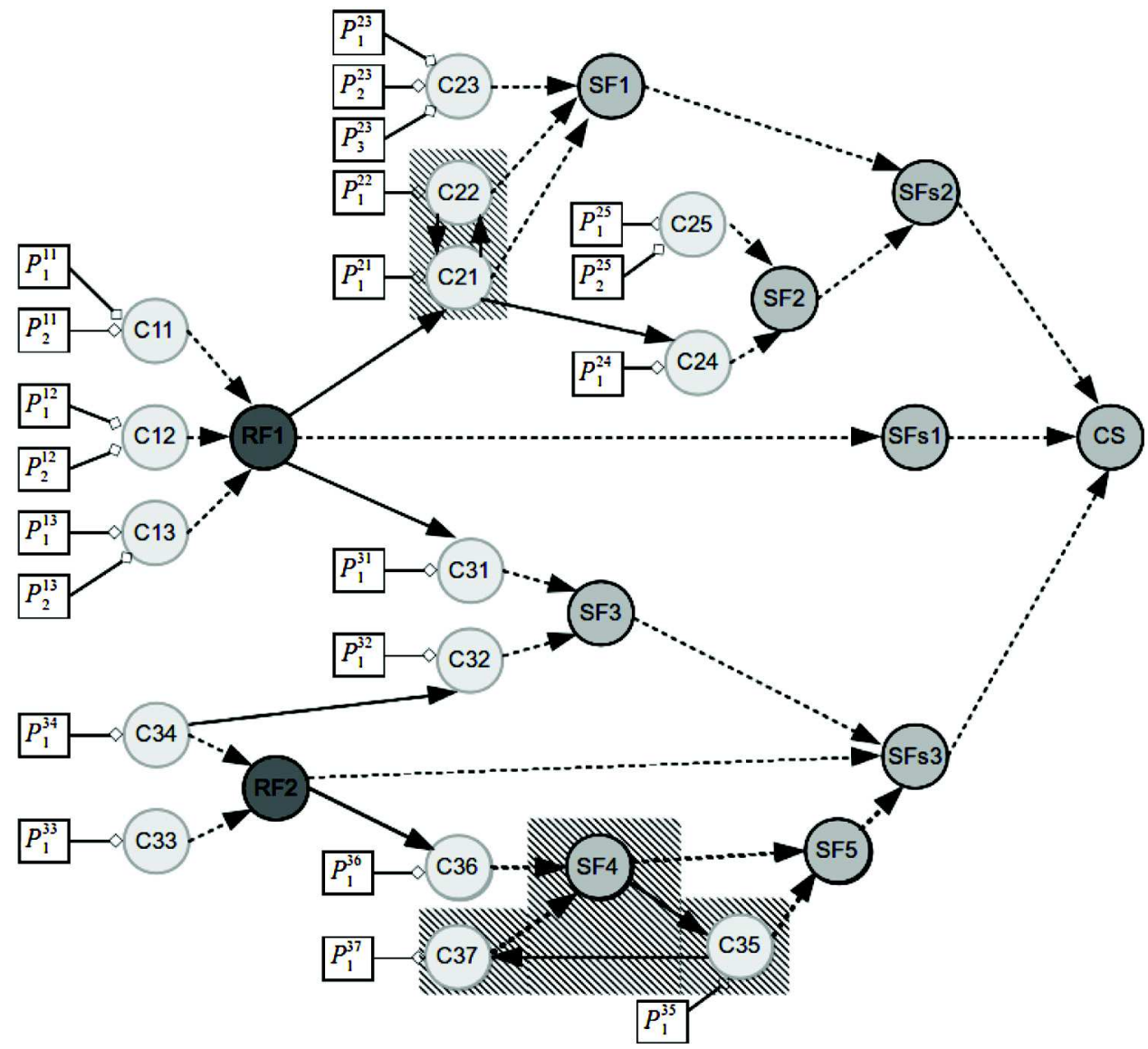

Fig. 11. Modeled fictitious multi-component system. 
If attributes $\lambda_{K O}$, of entities are beyond the maximum acceptable probability of inability to fulfill the planned productive tasks, the impact of the maintenance of the components, which are indicated by the attributes id of those same entities, can be checked $a$ priori. This is can be done by setting, for example, to zero (or to a very low value) the values of the local prognoses of the components that are supposed to undergo maintenance and by running the proposed generic function again with these new values of the local prognostics. Afterward, the function will provide the new values of the attributes $\lambda_{K O}, \lambda_{F}, \lambda_{O O}, \lambda_{L R}$ and $i d$ for the new proposed repairs if some attributes $\lambda_{K O}$ still have too high values. The discrepancies between the values of the attributes $\lambda_{K O}, \lambda_{F}, \lambda_{O O}, \lambda_{L R}$ before and after maintenance will so enable to assess the efficiency of the maintenance of components relatively to the ability of the entities (mainly functions) of the complex system to complete the planned tasks.

Thanks to the proposed decision indicators, production scheduling and maintenance management can jointly make the best decision according to joint performance indicators $[5,6,35]$. The decision can be, but not limited to, to validate the assignment of productive tasks, to reduce the number of assigned tasks, to change the assigned tasks, to schedule downtimes and, for downtimes, to choose the components to maintain.

\section{Example of multi-component system and experimental results}

The aim of this part is to present the decision support indicators the proposed generic function for complex system provides and how this function could be used by production and maintenance planning. These results are obtained from a fictitious multicomponent system whose goal is to bring together most of the situations that may be encountered in complex systems from the point of view of the proposed prognostic function computation. The fictitious complex system, which is presented in Fig. 10, is made of three subsystems that contains redundancy functions or simple functions. Several components (Cx) implement the functions. In Fig. 10, the arcs are the causal relationships of the structural knowledge modeling.

This fictitious multi-component system is modeled according to the behavioral, structural and functional knowledge modeling principles described in this paper. The model of this fictitious complex system is shown in Fig. 11 where $\mathrm{Cx}$ is for the component number $x, P_{i}^{x}$ is the local prognostics number $i$ of the component Cx, RFk is the redundancy function number $k, \mathrm{SFl}$ is the simple function number $l$, SFsj is the subsystem number $j$ that is considered as a simple functions and CS is the complex system.

Transforming the graph cycles, that the hatched areas highlight, of the modeled fictitious complex system by using functions of interdependence, the model of the fictitious complex system becomes the one presented in Fig. 12 where SFim is the simple function of interdependence number $m$. Then three graphs are generated to avoid to introduce several times the probability of the KO state of one node to the computation of the KO state of another one unless it belongs to a redundancy function. These graphs are presented in Figs. 13-15 on which the proposed generic is successively run. The results computed for all the nodes of the graph of Fig. 13 are stored. For the graph of Fig. 14, only the results

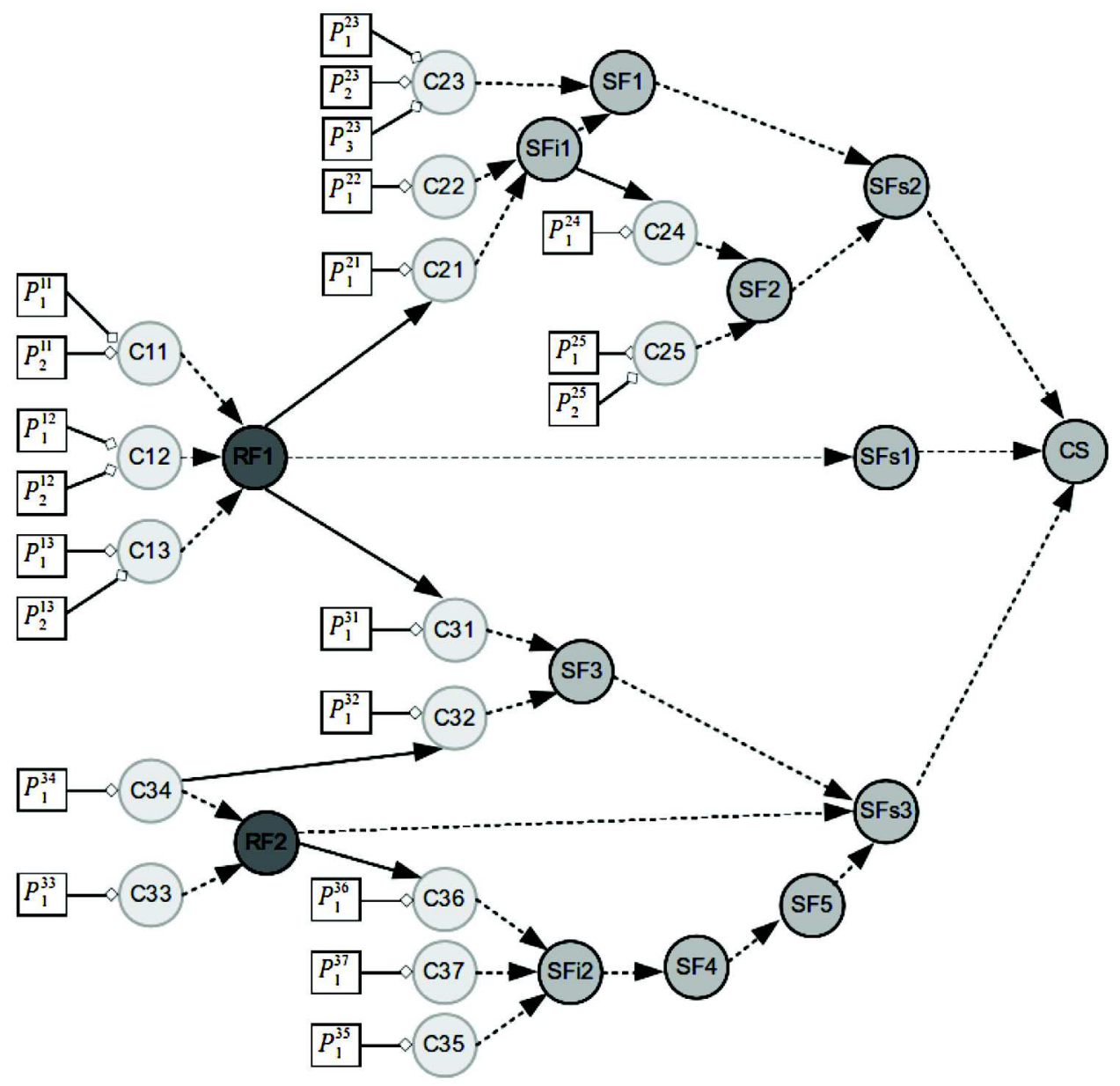

Fig. 12. Transformed model of the fictitious multi-component system to suppress graph cycles. 


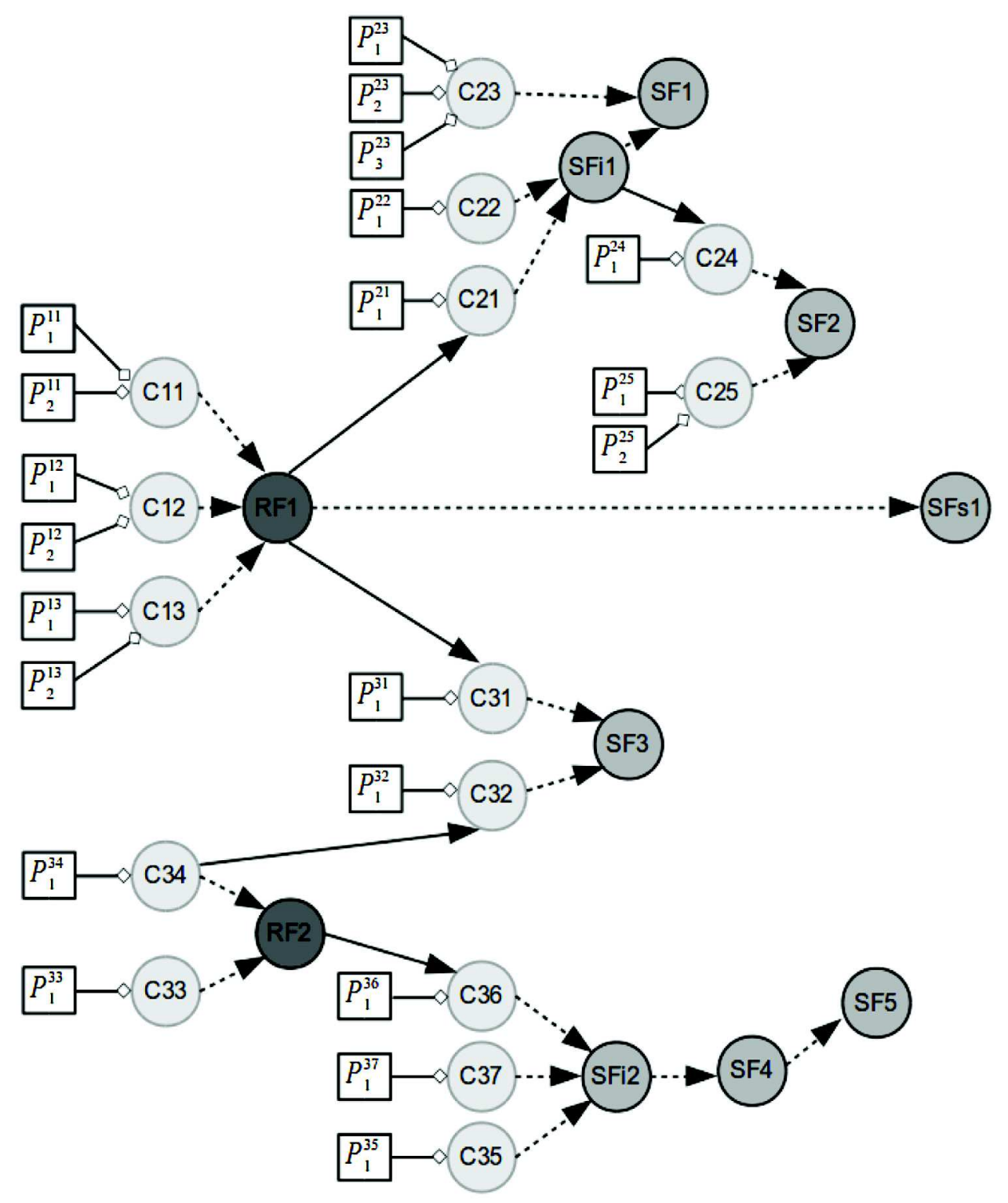

Fig. 13. First graph generated to avoid to introduce several times the probability of the KO state of one node.

computed for the nodes SFs2 and SFs3 are stored and, for the graph of Fig. 15, only the results computed for the node CS are stored. The stored values of the attributes of nodes are decision support indicators for production and maintenance scheduling.

Four scenarios whose results are presented in Table 1 have been computed from the graphs of Figs. 13-15. In those scenarios, we consider that the planned productive tasks will solicit all the functions of the system and that their probability to fail before the completion of those tasks must not be beyond $1.5 \mathrm{e}-2$ and that the only criterion to maintain components is the improvement of reliability according to rules (13) and (14).

The scenario 1 consists of a situation where all the local prognostics assess probabilities of failure before the end of scheduled tasks assigned to the complex system at $1 \mathrm{e}-4$.

The scenario 2 is based on the same situation as the scenario 1 but the local prognostics $P_{1}^{12}$ and $P_{1}^{25}$ provide assessed probabilities of failure before the end of scheduled tasks assigned to the system at $3 \mathrm{e}-2$ and the local prognostics $P_{2}^{11}, P_{1}^{21}, P_{1}^{34}$ and $P_{1}^{37}$ provide assessed probabilities of failure before the end of scheduled tasks assigned to the system at $1 \mathrm{e}-2$. Then two decisions can be made according to the indicators provided by the proposed generic function and presented in Table 1:

- The production planning defines a new sequence of tasks for which there is no function whose probability of failure before the achievement of this sequence is greater than $1.5 \mathrm{e}-2$, and maintenance planning schedules the necessary actions on components C25 and C37 after the completion of this sequence thanks to the values of their local prognostics.

- The production planning decides not to solicit the complex system and maintenance of components C25 and C37 is undertaken.

The scenario 3 is based on the same situation as the scenario 2 but the components whose identifiers appear in the attributes $i d$ of the functions whose attributes $\lambda_{K O}$ or $\lambda_{L R}$ are greater than $1.5 \mathrm{e}-2$ are maintained. These components are C25 and C37 highlighted in dark grey in Table 1 . To check if the maintenance of those two components is enough to get under the maximum allowed probability that a function of the system fails before the 


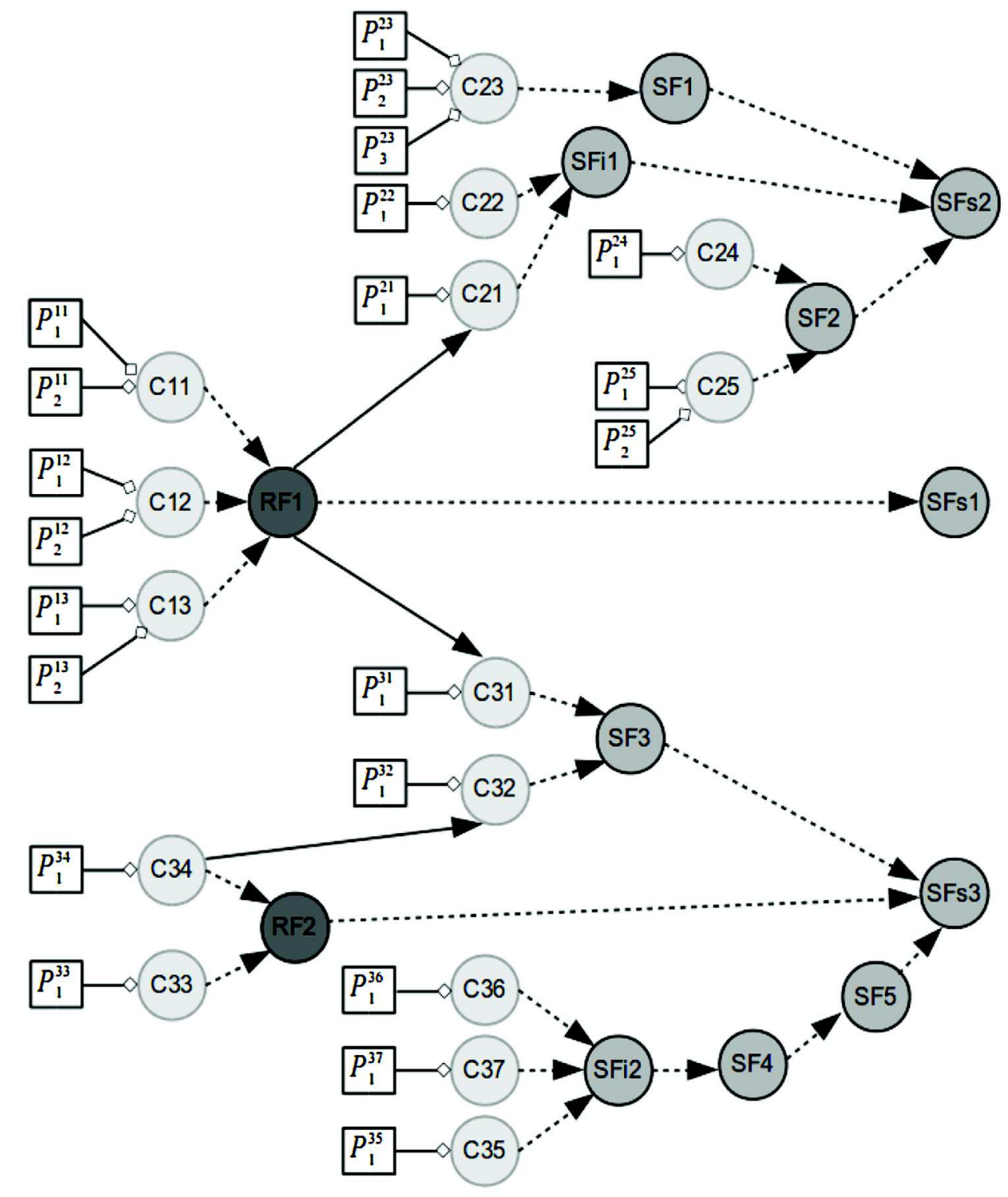

Fig. 14. Second graph generated to avoid to introduce several times the probability of the KO state of one node.

completion of the planned tasks, the local prognostics $P_{1}^{25}, P_{2}^{25}$ and $P_{1}^{37}$ are set to 1 e- 6 assuming the maintenance of C25 and C37 is done (but it could be other values much lower than the ones before their maintenance). For this scenario, the proposed generic function is successively run for the three graphs with those new values of $P_{1}^{25}, P_{2}^{25}$ and $P_{1}^{37}$. The indicators it provides suggests to maintain $\mathrm{C} 34$ too, if production planning needs that the maximum allowed probability of the complex system CS before the completion of the planned tasks is $1.5 \mathrm{e}-2$. Thus, three components should undergo maintenance to get under the maximum allowed probability of failure of a function before the end of the tasks planned for the scenario 2. After the maintenance of C34, we suppose $P_{1}^{34}$ is set to $1 \mathrm{e}-6$ too (but it could be another value much lower than the one before its maintenance) and then the proposed function is processed again for the three graphs. The provided indicators show that if C25, C37 and C34 are maintained, there is no more function whose probability of failure before the completion of the planned tasks is greater than 1.5e-2 (the supposed maximum allowed threshold). Indeed, in this case $\lambda_{K O}^{C S}$ is highest value which is lower than $1.09 \mathrm{e}-2$.
The scenario 4 highlights the ability of the proposed generic function to predict propagations of failures and their effects on the functioning of functions and subsystems. In this case the systems must implement a diagnostic module. When a local diagnostic states that a component is failed, at least one of its local prognostics must be set to one (the value of the prognostic must be saved in a buffer to be recovered if needed later). Then the prognostic function is successively run from step 4 for the three graphs. Then, each component or function whose attribute $\lambda_{K O}$ is equal to one can consequently be considered out of order. These results can especially be used for the consistency based diagnosis approach in order to reduce the number of candidate components [2]. Indeed, the second stage of consistency based diagnostic consists in verifying the candidates. If components or functions that are prognosed "out of order" consequently to the failure of a component are still operating according to their own diagnostic modules, one can consider that the component that is the origin of these prognostics is not failed and that it was due to a false detection and so the local prognostic that was set to one must be reset to the value that was saved in the buffer and the proposed generic function is processed again for the three graphs. The 


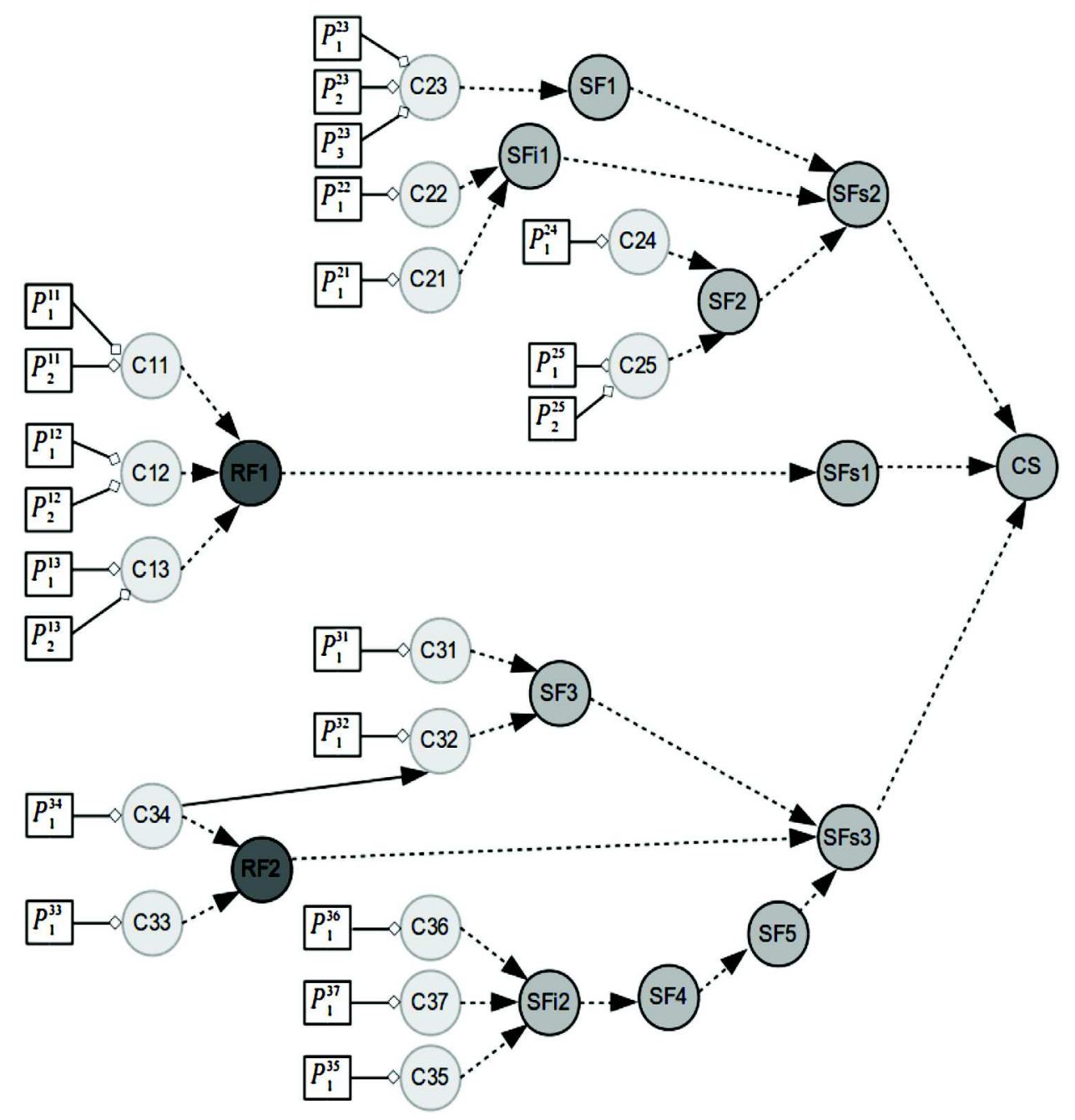

Fig. 15. Third graph generated to avoid to introduce several times the probability of the KO state of one node.

computed values of $\lambda_{K O}$ and $\lambda_{L R}$ can also be used by production operators to make decision about how to adapt to the detected failure. The scenario 4 is based on the scenario 3 but the components $\mathrm{C} 12$ and $\mathrm{C} 34$ are diagnosed as failed. The consequences are that functions SF3, SFs3 and CS are out of order and the redundancy of RF2 is lost.

\section{Conclusion}

A generic function providing decision supports for production and maintenance based on Bayesian networks to infer the ability of complex system to complete planned tasks from local prognostics and on an extension to identify components to be maintained, was presented in this paper. The decision support indicators it provides help the production planning to assign productive tasks and also to guide maintenance toward the components that should firstly be maintained. The implementation of this function requires a modeling of the system that consists of graphs that represent functional, structural and a part of the behavioral knowledge about the system. The method to build those modeling graphs to adapt them to the generic function requirements was presented. The inputs of this function are the local prognostics of the components. These local prognostics provide the probabilities that the components fail according to their different failures modes before the end of the tasks that production planning has assigned to the system. From these local prognostics, the generic function assesses the values of the transitions between the states of each node of the complex system model and so it provides, for all the hierarchical levels, the ability of functions and subsystems to fulfill the planned productive tasks. These values are the probabilities that the different entities of the complex systems (components, functions, subsystems, etc.) fail or become out of order before the end of the assigned tasks. These probabilities are decision support indicators used by production planning to valid or not to valid the tasks scheduling for a given complex system and they can be used to define the downtimes of this given complex system to perform maintenance. The proposed prognostic function also guides maintenance toward the components that need it in order to shorten downtimes by enabling to plan the maintenance actions and their logistics in advance. Therefore the proposed generic function contributes to a better compromise between the satisfaction of the respective objectives of the condition based maintenance and of the production planning. The proposed prognostic function can predict propagations of failures and their effects on the functioning of functions and subsystems and thus it can be reused to implement a consistency based diagnostic function dedicated to the system. In this case the diagnostic function and the prognostic function can share a part of the system modeling. Scenarios were described to show how the provided decision support indicators can be used for production and maintenance planning purposes. 
Table 1

Results of the computed scenarios for the fictitious multi-component system.

\begin{tabular}{|c|c|c|c|c|c|c|c|c|c|c|c|c|}
\hline \multirow[b]{2}{*}{ Node id } & \multicolumn{3}{|l|}{ Scenario 1} & \multicolumn{3}{|l|}{ Scenario 2} & \multicolumn{3}{|l|}{ Scenario 3} & \multicolumn{3}{|l|}{ Scenario 4} \\
\hline & $\lambda_{L R}$ & $\lambda_{K O}$ & id & $\lambda_{L R}$ & $\lambda_{K O}$ & id & $\lambda_{L R}$ & $\lambda_{K O}$ & id & $\lambda_{L R}$ & $\lambda_{K O}$ & id \\
\hline CS & & $1.40 \mathrm{E}-03$ & $\mathrm{C} 23$ & & $5.98 \mathrm{E}-02$ & $\mathrm{C} 25$ & & $2.08 \mathrm{E}-02$ & C34 & & $1.00 \mathrm{E}+00$ & C34 \\
\hline SFi2 & & $3.00 \mathrm{E}-04$ & $\mathrm{C} 22$ & & $1.02 \mathrm{E}-02$ & C37 & & $2.02 \mathrm{E}-04$ & C35 & & $3.01 \mathrm{E}-04$ & C35 \\
\hline SFi1 & & $2.00 \mathrm{E}-04$ & $\mathrm{C} 21$ & & $1.01 \mathrm{E}-02$ & C21 & & $1.01 \mathrm{E}-02$ & $\mathrm{C} 21$ & & $1.01 \mathrm{E}-02$ & $\mathrm{C} 21$ \\
\hline SFs3 & & $6.00 \mathrm{E}-04$ & C35 & & $2.03 \mathrm{E}-02$ & C37 & & $1.04 \mathrm{E}-02$ & C34 & & $1.00 \mathrm{E}+00$ & C34 \\
\hline SFs2 & & $8.00 \mathrm{E}-04$ & $\mathrm{C} 23$ & & $4.03 \mathrm{E}-02$ & C25 & & $1.05 E-02$ & $\mathrm{C} 21$ & & $1.05 \mathrm{E}-02$ & C21 \\
\hline SFs1 & & $8.00 \mathrm{E}-12$ & C13 & & $6.08 \mathrm{E}-08$ & $\mathrm{C} 12$ & & $6.08 \mathrm{E}-08$ & C12 & & $2.02 \mathrm{E}-06$ & C12 \\
\hline SF5 & & $3.00 \mathrm{E}-04$ & C35 & & $1.02 \mathrm{E}-02$ & C37 & & $2.02 \mathrm{E}-04$ & C35 & & $3.01 \mathrm{E}-04$ & C35 \\
\hline SF4 & & $3.00 \mathrm{E}-04$ & C35 & & $1.02 \mathrm{E}-02$ & C37 & & $2.02 \mathrm{E}-04$ & C35 & & $3.01 \mathrm{E}-04$ & C35 \\
\hline SF3 & & $3.00 \mathrm{E}-04$ & C32 & & $1.02 \mathrm{E}-02$ & C34 & & $1.02 \mathrm{E}-02$ & C34 & & $1.00 \mathrm{E}+00$ & C34 \\
\hline SF2 & & $5.00 \mathrm{E}-04$ & $\mathrm{C} 25$ & & $4.00 \mathrm{E}-02$ & $\mathrm{C} 25$ & & $1.02 \mathrm{E}-02$ & $\mathrm{C} 21$ & & $1.02 \mathrm{E}-02$ & $\mathrm{C} 21$ \\
\hline SF1 & & $5.00 \mathrm{E}-04$ & $\mathrm{C} 23$ & & $1.04 \mathrm{E}-02$ & $\mathrm{C} 21$ & & $1.04 \mathrm{E}-02$ & $\mathrm{C} 21$ & & $1.04 \mathrm{E}-02$ & $\mathrm{C} 21$ \\
\hline RF2 & $2.00 \mathrm{E}-04$ & $1.00 \mathrm{E}-08$ & C33 & $1.01 \mathrm{E}-02$ & $1.00 \mathrm{E}-06$ & C34 & $1.01 E-02$ & $1.00 \mathrm{E}-06$ & C34 & $1.00 \mathrm{E}+00$ & $1.00 \mathrm{E}-04$ & C34 \\
\hline RF1 & $1.20 \mathrm{E}-07$ & $8.00 \mathrm{E}-12$ & $\mathrm{C} 13$ & $3.12 \mathrm{E}-04$ & $6.08 \mathrm{E}-08$ & C12 & $3.12 \mathrm{E}-04$ & $6.08 \mathrm{E}-08$ & C13 & $1.03 \mathrm{E}-02$ & $2.02 \mathrm{E}-06$ & C12 \\
\hline C37 & & $1.00 \mathrm{E}-04$ & C37 & & $1.00 \mathrm{E}-02$ & C37 & & $1.00 \mathrm{E}-06$ & C37 & & $1.00 \mathrm{E}-06$ & C37 \\
\hline C36 & & $1.00 \mathrm{E}-04$ & C36 & & $1.01 \mathrm{E}-04$ & C36 & & $1.01 \mathrm{E}-04$ & C36 & & $2.00 \mathrm{E}-04$ & C36 \\
\hline C35 & & $1.00 \mathrm{E}-04$ & C35 & & $1.00 \mathrm{E}-04$ & C35 & & $1.00 \mathrm{E}-04$ & C35 & & $2.00 \mathrm{E}-04$ & C35 \\
\hline C34 & & $1.00 \mathrm{E}-04$ & C34 & & $1.00 \mathrm{E}-02$ & C34 & & $1.00 \mathrm{E}-02$ & C34 & & $1.00 \mathrm{E}+00$ & C34 \\
\hline C33 & & $1.00 \mathrm{E}-04$ & C33 & & $1.00 \mathrm{E}-04$ & C33 & & $1.00 \mathrm{E}-04$ & C33 & & $1.00 \mathrm{E}-04$ & C33 \\
\hline C32 & & $2.00 \mathrm{E}-04$ & C32 & & $1.01 \mathrm{E}-02$ & C34 & & $1.01 \mathrm{E}-02$ & C34 & & $1.00 \mathrm{E}+00$ & C34 \\
\hline C31 & & $1.00 E-04$ & C31 & & $1.00 \mathrm{E}-04$ & C31 & & $1.00 \mathrm{E}-04$ & C31 & & $1.02 \mathrm{E}-04$ & C31 \\
\hline $\mathrm{C} 25$ & & $2.00 \mathrm{E}-04$ & $\mathrm{C} 25$ & & $3.01 \mathrm{E}-02$ & $\mathrm{C} 25$ & & $2.00 \mathrm{E}-06$ & $\mathrm{C} 25$ & & $2.00 \mathrm{E}-06$ & $\mathrm{C} 25$ \\
\hline $\mathrm{C} 24$ & & $3.00 \mathrm{E}-04$ & $\mathrm{C} 24$ & & $1.02 \mathrm{E}-02$ & $\mathrm{C} 21$ & & $1.02 \mathrm{E}-02$ & $\mathrm{C} 21$ & & $1.02 \mathrm{E}-02$ & $\mathrm{C} 21$ \\
\hline $\mathrm{C} 23$ & & $3.00 \mathrm{E}-04$ & $\mathrm{C} 23$ & & $3.00 \mathrm{E}-04$ & $\mathrm{C} 23$ & & $3.00 \mathrm{E}-04$ & $\mathrm{C} 23$ & & $3.00 \mathrm{E}-04$ & $\mathrm{C} 23$ \\
\hline $\mathrm{C} 22$ & & $1.00 \mathrm{E}-04$ & $\mathrm{C} 22$ & & $1.00 \mathrm{E}-04$ & $\mathrm{C} 22$ & & $1.00 \mathrm{E}-04$ & $\mathrm{C} 22$ & & $1.00 \mathrm{E}-04$ & $\mathrm{C} 22$ \\
\hline $\mathrm{C} 21$ & & $1.00 \mathrm{E}-04$ & $\mathrm{C} 21$ & & $1.00 \mathrm{E}-02$ & $\mathrm{C} 21$ & & $1.00 \mathrm{E}-02$ & $\mathrm{C} 21$ & & $1.00 \mathrm{E}-02$ & $\mathrm{C} 21$ \\
\hline C13 & & $2.00 \mathrm{E}-04$ & C13 & & $2.00 \mathrm{E}-04$ & $\mathrm{C} 13$ & & $2.00 \mathrm{E}-04$ & $\mathrm{C} 13$ & & $2.00 \mathrm{E}-04$ & $\mathrm{C} 13$ \\
\hline $\mathrm{C} 12$ & & $2.00 \mathrm{E}-04$ & $\mathrm{C} 12$ & & $3.01 \mathrm{E}-02$ & $\mathrm{C} 12$ & & $3.01 \mathrm{E}-02$ & $\mathrm{C} 12$ & & $1.00 \mathrm{E}+00$ & C12 \\
\hline C11 & & $2.00 \mathrm{E}-04$ & $\mathrm{C} 11$ & & $1.01 \mathrm{E}-02$ & C11 & & $1.01 \mathrm{E}-02$ & C11 & & $1.01 \mathrm{E}-02$ & C11 \\
\hline
\end{tabular}

Further developments of this work will deal with the implementation of the prognostic function on a real system. Other developments will aim at making interoperable various uncertainty models used for the local prognoses. This could be brought into operation thanks to the Dempster-Shafer evidence theory or of the transferable belief model that includes a credal level to represent and to combine the information and a pignistic level for decision making [13,38-40].

Appendix A.

$\lambda_{K O}^{C X}(t)=1-\left(1-\lambda_{F}^{C X}(t)\right)\left(1-\lambda_{O O}^{C X}(t)\right)$

$\lambda_{K O}^{C X}(t)=1-\left(1-\lambda_{F}^{C X}(t)\right)\left\{1-\left[1-\Pi_{E i \in \Gamma^{-1}(C x)}\left(1-\lambda_{K O}^{E i}(t)\right)\right]\right\}$

$\lambda_{K O}^{C x}(t)=1-\left(1-\lambda_{F}^{C x}(t)\right) \cdot \Pi_{E i \in \Gamma^{-1}(C x)}\left(1-\lambda_{K O}^{E i}(t)\right)$

If a node $\mathrm{E}$ is a simple function, then $\lambda_{F}^{E}(t)=0$, hence:

$$
\begin{aligned}
\lambda_{K O}^{C X}(t) & =1 \\
& -\left(1-\lambda_{F}^{C x}(t)\right) \cdot \Pi_{E i \in \Gamma^{-1}(C x)}\left\{1-\left[1-\left(1-\lambda_{F}^{E i}(t)\right)\left(1-\lambda_{O O}^{E i}(t)\right)\right]\right\} \\
\lambda_{K O}^{C X}(t) & =1-\left(1-\lambda_{F}^{C x}(t)\right) \cdot \Pi_{E i \in \Gamma^{-1}(C x)}\left[\left(1-\lambda_{F}^{E i}(t)\right)\left(1-\lambda_{O O}^{E i}(t)\right)\right] \\
\lambda_{K O}^{C X}(t) & =1-\left(1-\lambda_{F}^{C x}(t)\right) \cdot \Pi_{E i \in \Gamma^{-1}(C x)} \\
& \left\{\left(1-\lambda_{F}^{E i}(t)\right)\left[1-\left[1-\Pi_{E j \in \Gamma^{-1}(E i)}\left(1-\lambda_{K O}^{E j}(t)\right)\right]\right]\right\}
\end{aligned}
$$

$$
\begin{aligned}
\lambda_{K O}^{C X}(t) & =1-\left(1-\lambda_{F}^{C x}(t)\right) \cdot \Pi_{E i \in \Gamma^{-1}(C x)} \\
& \left\{\left(1-\lambda_{F}^{E i}(t)\right) \cdot \Pi_{E j \in \Gamma^{-1}(E i)}\left(1-\lambda_{K O}^{E j}(t)\right)\right\}
\end{aligned}
$$

If we note $A^{-1}(E)$ the set of the nodes, from which the node $\mathrm{E}$ is attainable, we then recursively obtain:

$\lambda_{K O}^{C x}(t)=1-\left(1-\lambda_{F}^{C x}(t)\right) \cdot \Pi_{E k \in \mathrm{A}^{-1}(C x)}\left(1-\lambda_{F}^{E k}(t)\right)$

Hence, the value $\left.\left.\lambda_{F}^{E m}(t) \in\right] 0,1\right]$, if it was zero, would decrease at most $\lambda_{K O}^{C X}(t)$ is such as:

$\left.\lambda_{F}^{E m}(t)=\max \lambda_{F}^{C x}(t), \max _{E k \in A^{-1}(C x)}\left(\lambda_{F}^{E k}(t)\right)\right)$

\section{References}

[1] A. Arnaiz, S. Ferreiro, M. Buderath, New decision support system based on operational risk assessment to improve aircraft operability, Proc. Inst. Mech. Eng. Part O J. Risk Reliab. 224 (2010) 137-147.

[2] J. Biteus, M. Nybergb, E. Friska, An algorithm for computing the diagnoses with minimal cardinality in a distributed system, Eng. Appl. Artif. Intell. 21 (2008) 269-276.

[3] M. Blaha, J. Rumbaugh, Object Oriented Modeling and Design with UML, 2nd ed., Pearson Prentice-Hall, Chesterfield, 2005.

[4] C. Byington, M. Watson, M. Roemer, T. Galie, Prognostic enhancements to gas turbine diagnostic systems, Proceedings of the IEEE Aerospace Conference, Big Sky, USA, 2001.

[5] M. Chaouqi, J. Benhra, A. Zakari, Agile approach for joint scheduling of production and maintenance in flow shop, Int. J. Comput. Appl. 59 (2012) 2936.

[6] T. Coudert, B. Grabot, B. Archimède, Production/maintenance co-operative scheduling using multi-agents and fuzzy logic, Int. J. Prod. Res. 40 (2002) 4611 4632.

[7] J. Dunjo, V. Fthenakis, J.A. Vilchez, J. Arnaldos, Hazard and operability (HAZOP) analysis. A literature review, J. Hazard. Mater. 173 (2010) 19-32.

[8] W. Elghazel, J. Bahi, C. Guyeux, M. Hakem, K. Medjaher, N. Zerhouni, Dependability of wireless sensor networks for industrial prognostics and health management, Comput. Ind. 68 (2015) 1-15. 
[9] Q. Feng, Y. Chen, B. Sun, S. Li, An optimization method for condition based maintenance of aircraft fleet considering prognostics uncertainty, Sci. World J. 2014 (2014), doi:http://dx.doi.org/10.1155/2014/430190 (Article ID 430190).

[10] S. Ferreiro, A. Arnaiz, B. Sierra, I. Irigoien, Application of Bayesian networks in prognostics for a new integrated vehicle health management concept, Expert Syst. Appl. 39 (2012) 6402-6418.

[11] P. Goupil, AIRBUS state of the art and practices on FDI and FTC in flight control system, Control Eng. Pract. 19 (2011) 524-539.

[12] R. Gouriveau, N. Zerhouni, Connexionist-systems-based long term prediction approaches for prognostics, IEEE Trans. Reliab. 61 (2012) 909-920.

[13] W. He, N. Williard, M. Osterman, M. Pecht, Prognostics of lithium-ion batteries based on Dempster-Shafer theory and the Bayesian Monte Carlo method, J. Power Sources 196 (2011) 10314-10321.

[14] A. Heng, A.C.C. Tan, J. Mathew, N. Montgomery, D. Banjevic, A.K.S. Jardine, Intelligent condition-based prediction of machinery reliability, Mech. Syst. Sig. Process. 23 (2009) 1600-1614.

[15] W. Hong-Feng, Prognostics and Health Management for complex system based on fusion of model-based approach and data-driven approach, Phys. Procedia 24 (2012) 828-831.

[16] B. Iung, M. Monnin, A. Voisin, P. Cocheteux, E. Levrat, Degradation state modelbased prognosis for proactively maintaining product performance, CIRP Ann. Manuf. Technol. 57 (2008) 49-52.

[17] G. Jacazio, M. Sorli, D. Bolognese, D. Ferrara, Health management system for the pantographs of tilting trains, Proceedings of First European Conference of the Prognostics and Health Management Society, Dresden, Germany, 2012, pp. 127-140 (July 3-5).

[18] A.K.S. Jardine, D. Lin, D. Banjevic, A review on machinery diagnostics and prognostics implementing condition-based maintenance, Mech. Syst. Sig. Process. 20 (2006) 1483-1510.

[19] G. Jin, D.E. Matthews, Z. Zhou, A Bayesian framework for on-line degradation assessment and residual life prediction of secondary batteries in spacecraft, Reliab. Eng. Syst. Saf. 113 (2013) 7-20.

[20] N. Julka, A. Thirunavukkarasu, P. Lendermann, B.P. Gan, A. Schirrmann, H. Fromm, E. Wong, Making use of prognostics health management information for aerospace spare components logistics network optimization, Comput. Ind. 62 (2011) 613-622.

[21] T. Koski, J.M. Noble, Bayesian Networks: an Introduction, John Wiley \& Sons Inc., Chichester, 2009.

[22] A. Kossiakoff, W.N. Sweet, S. Seymour, S.M. Biemer, Systems Engineering Principles and Practice, 2nd ed., John Wiley \& Sons Inc., Hoboken, 2011.

[23] K. Le Son, M. Fouladirad, A. Barros, E. Levrat, B. Iung, Remaining useful life estimation based on stochastic deterioration models: a comparative study, Reliab. Eng. Syst. Saf. 112 (2013) 165-175.

[24] J. Lee, J. Ni, D. Djurdjanovic, H. Qiu, H. Liao, Intelligent prognostics tools and emaintenance, Comput. Ind. 57 (2006) 476-489.

[25] Q. Liu, M. Dong, W. Lv, X. Genga, Y. Li, A novel method using adaptive hidden semi-Markov model for multi-sensor monitoring equipment health prognosis, Mech. Syst. Sig. Process. 64-65 (2015) 217-232.

[26] G. Medina-Oliva, P. Weber, B. Iung, PRM-based patterns for knowledge formalisation of industrial systems to support maintenance strategies assessment, Reliab. Eng. Syst. Saf. 116 (2013) 38-56.

[27] G. Medina-Oliva, P. Weber, B. Iung, Industrial system knowledge formalization to aid decision making in maintenance strategies assessment, Eng. Appl. Artif. Intell. 37 (2015) 343-360.

[28] K. Medjaher, D.A. Tobon-Mejia, N. Zerhouni, Remaining useful life estimation of critical components with application to bearings, IEEE Trans. Reliab. 61 (2012) 292-302.
[29] A. Muller, M.C. Suhner, B. Iung, Formalisation of a new prognosis model for supporting proactive maintenance implementation on industrial system, Reliab. Eng. Syst. Saf. 93 (2008) 234-253.

[30] K.A. Nguyen, P. Do, A. Grall, Multi-level predictive maintenance for multicomponent systems, Reliab. Eng. Syst. Saf. 144 (2015) 83-94.

[31] L. Rémy, A. Alam, N. Haddar, A. Köster, N. Marchal, Growth of small cracks and prediction of lifetime in high-temperature alloys, Mater. Sci. Eng. A 468-470 (2007) 40-50.

[32] S. Sankararaman, M.J. Daigle, K. Goebel, Uncertainty quantification in remaining useful life prediction using first-order reliability methods, IEEE Trans. Reliab. 63 (2014) 603-619.

[33] S. Sankararaman, Y. Ling, S. Mahadevan, Uncertainty quantification and model validation of fatigue crack growth prediction, Eng. Fract. Mech. 78 (2011) $1487-1504$.

[34] A. Saxena, J. Celaya, B. Saha, S. Saha, K. Goebel, Metrics for offline evaluation of prognostic performance, Int. J. Prognostics Health Manage. 1 (2010) 1-20.

[35] E. Sanmarti, A. Espufia, L. Puigjaner, Batch production and preventive maintenance scheduling under equipment failure uncertainty, Comput. Chem. Eng. 21 (1997) 1157-1158.

[36] P. Scarf, A Framework for condition monitoring and condition based maintenance, Qual. Technol. Quant. Manage. 4 (2007) 301-312.

[37] E.L. Silva Teixeira, B. Tjahjono, S.C. Absi Alfaro, A novel framework to link prognostics and health management and product-service systems using online simulation, Comput. Ind. 63 (2012) 669-679.

[38] C. Simon, P. Weber, E. Levrat, Bayesian networks and evidence theory to model complex systems reliability, J. Comput. 2 (2007) 33-43.

[39] C. Simon, P. Weber, A. Evsukoff, Bayesian networks inference algorithm to implement Dempster Shafer theory in reliability analysis, Reliab. Eng. Syst. Saf. 93 (2008) 950-963.

[40] P. Smets, R. Kennes, The transferable belief model, Artif. Intell. 66 (1994) 191234.

[41] R. Sweeney, Achieving Service-Oriented Architecture: Applying an Enterprise Architecture Approach, John Wiley \& Sons Inc., Hoboken, 2010.

[42] R.E. Tarjan, Depth-first search and linear graph algorithms, SIAM J. Comput. 1 (1972) 146-160, doi:http://dx.doi.org/10.1137/0201010.

[43] D.A. Tobon-Mejia, K. Medjaher, N. Zerhouni, G. Tripot, A data-driven failure prognostics method based on mixture of Gaussians hidden Markov models, IEEE Trans. Reliab, 61 (2012) 491-503.

[44] G. Vachtsevanos, F.L. Lewis, M. Roemer, A. Hess, B. Wu, Intelligent Fault Diagnosis and Prognosis for Engineering System, John Wiley \& Sons Inc., Hoboken, 2006.

[45] A. Voisin, E. Levrat, P. Cocheteux, B. Iung, Generic prognosis model for proactive maintenance decision support: application to pre-industrial emaintenance test bed, J. Intell. Manuf. 21 (2010) 177-193.

[46] P. Weber, L. Jouffe, Complex system reliability modelling with dynamic Object Oriented Bayesian Networks (DOOBN), Reliab. Eng. Syst. Saf. 91 (2006) 149162.

[47] H. Worn, T. Langle, M. Albert, A. Kazi, A. Brighenti, S. Revuelta Seijo, Diamond: distributed multi-agent architecture for monitoring and diagnosis, Prod. Plann. Control 15 (2004) 189-200.

[48] R.C.M. Yam, P.W. Tse, L. Li, P. Tu, Intelligent predictive decision support system for condition-based maintenance, Int. J. Adv. Manuf. Technol. 17 (2001) 383391.

[49] F. Zhao, Z. Tian, Y. Zeng, Uncertainty quantification in gear remaining useful life prediction through an integrated prognostics method, IEEE Trans. Reliab. 62 (2013) 146-159.

[50] E. Zio, Reliability engineering: old problems and new challenges, Reliab. Eng. Syst. Saf. 94 (2009) 125-141. 